\title{
Review of the speculative role of co-infections in Streptococcus suis-associated diseases in pigs
}

Milan R. Obradovic ${ }^{1}$, Mariela Segura', Joaquim Segalés ${ }^{2,3,4}$ and Marcelo Gottschalk ${ }^{1 *}$ (D)

\begin{abstract}
Streptococcus suis is one of the most important bacterial swine pathogens affecting post-weaned piglets, causing mainly meningitis, arthritis and sudden death. It not only results in severe economic losses but also raises concerns over animal welfare and antimicrobial resistance and remains an important zoonotic agent in some countries. The definition and diagnosis of S. suis-associated diseases can be complex. Should S. suis be considered a primary or secondary pathogen? The situation is further complicated when referring to respiratory disease, since the pathogen has historically been considered as a secondary pathogen within the porcine respiratory disease complex (PRDC). Is S. suis a respiratory or strictly systemic pathogen? S. suis is a normal inhabitant of the upper respiratory tract, and the presence of potentially virulent strains alone does not guarantee the appearance of clinical signs. Within this unclear context, it has been largely proposed that co-infection with some viral and bacterial pathogens can significantly influence the severity of S. suis-associated diseases and may be the key to understanding how the infection behaves in the field. In this review, we critically addressed studies reporting an epidemiological link (mixed infections or presence of more than one pathogen at the same time), as well as in vitro and in vivo studies of co-infection of $S$. suis with other pathogens and discussed their limitations and possibilities for improvement and proposed recommendations for future studies.
\end{abstract}

Keywords: Streptococcus suis, Co-infections in pigs, Mixed infections, Porcine reproductive and respiratory syndrome virus, Swine influenza virus, Porcine circovirus 2, Bacterial mixed and co-infections, Porcine respiratory disease complex

\section{Table of Contents}

1 Introduction

2 Complexity of the transition from infection (or colonization) to clinical disease caused by S. suis

3 Should S. suis be considered a respiratory or systemic pathogen? Or both?

4. S. suis and viral mixed and/or co-infections

\footnotetext{
*Correspondence: marcelo.gottschalk@umontreal.ca

1 Groupe de Recherche Sur Les Maladies Infectieuses en Production Animale (GREMIP), Centre de Recherche en Infectiologie Porcine et Aviaire (CRIPA), Faculty of Veterinary Medicine, University of Montreal, 3200 Sicotte, Saint-Hyacinthe, QC J2S 2M2, Canada

Full list of author information is available at the end of the article
}

4.1 S. suis and PRRSV mixed and co-infections

4.1.1 PRRSV-S. suis mixed infections

4.1.2 In vitro studies on the interactions between $\mathrm{S}$. suis and PRRSV

4.1.3 In vivo studies on the interactions between $S$. suis and PRRSV

4.2 S. suis and swine influenza type A virus (SIV) mixed and co-infections

4.2.1 SIV-S. suis mixed infections

4.2.2 In vitro studies on the interaction between $\mathrm{S}$. suis and SIV

4.2.3 In vivo studies on the interaction between S. suis and SIV

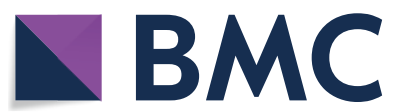

(c) The Author(s) 2021. This article is licensed under a Creative Commons Attribution 4.0 International License, which permits use, sharing, adaptation, distribution and reproduction in any medium or format, as long as you give appropriate credit to the original author(s) and the source, provide a link to the Creative Commons licence, and indicate if changes were made. The images or other third party material in this article are included in the article's Creative Commons licence, unless indicated otherwise in a credit line to the material. If material is not included in the article's Creative Commons licence and your intended use is not permitted by statutory regulation or exceeds the permitted use, you will need to obtain permission directly from the copyright holder. To view a copy of this licence, visit http://creativeco mmons.org/licenses/by/4.0/. The Creative Commons Public Domain Dedication waiver (http://creativecommons.org/publicdomain/ zero/1.0/) applies to the data made available in this article, unless otherwise stated in a credit line to the data. 
4.3 S. suis and porcine circovirus 2 mixed and co-infections

\subsubsection{PCV-2-S. suis mixed infections}

4.3.2 In vitro studies on the interaction between $S$. suis and PCV-2

4.3.3 In vivo studies on the interaction between $\mathrm{S}$. suis and PCV-2

4.4 S. suis and other virus co-infections

5 S. suis mixed and/or co-infections with other bacterial pathogens

5.1 S. suis mixed infections with other bacterial pathogens

5.2 In vitro and in vivo studies on the interaction between S. suis and other bacterial pathogens

6 Limitations of $\boldsymbol{S}$. suis co-infection studies

7 Conclusion and future perspectives

References

\section{Introduction}

Streptococcus suis is an important bacterial pathogen of swine with a worldwide distribution [1]. The most common clinical and pathological outcomes of the infection are meningitis, arthritis, endocarditis, septicemia and sudden death [2]. The definition of Streptococcus suisassociated diseases should be based on the combination of the presence of clinical signs, gross and/or microscopic lesions and bacterial isolation (in either predominance or pure culture) in affected organs and/or tissues. However, even after bacterial isolation, interpretation of the role of S. suis as a primary pathogen is not always easy. Furthermore, since diagnosis in the field may sometimes be based solely on clinical observations, differentiation with infections caused by other pathogens (such as Glaesserella parasuis) is difficult. The more specific role of S. suis in pneumonia is also a topic of debate. Clinical signs and mortality are mainly observed in weaned and (rarely) in suckling piglets and much less commonly in grower-finisher pigs. The infection caused by the pathogen not only results in severe economic losses but also raises animal welfare concerns. In addition, S. suis-associated diseases are difficult to control [2]. Even when the carrier rate of $S$. suis is high, the incidence of the disease varies from period to period and is usually less than $5 \%$ [2]. However, this is usually the case when antimicrobials (if allowed) are used as prophylactic/metaphylactic measures. One of the main problems is that antimicrobials that have efficacy are those the industry is trying to reduce given their importance in both human and veterinary medicine [2]. Recent data on antimicrobial susceptibility of S. suis are alarming. High rates of resistance to macrolides/lincosamides and tetracyclines are observed and attributed to the intensive use of antimicrobials in pigs [3]. S. suis is considered a niche for antimicrobial resistance and represents a high risk of transmission of such resistance to other veterinary and human pathogens due to the presence of mobile genetic elements carrying resistance genes transferable at high frequency within the species and, even more alarmingly, toward other bacterial species [3, 4]. S. suis is also considered an important zoonotic infection. The target populations are mainly workers in the swine and pork industry (Western countries), the general population due to close contact with pigs (China and other Asian countries) and individuals who consume raw or undercooked pork or pork by-products (Vietnam, Thailand and Laos) $[5,6]$.

S. suis had originally been classified into 35 serotypes based on the antigenicity of the capsular polysaccharide, which is suggested to be a major virulent factor [7]. However, six of the serotypes (serotypes 20,22, 26, 32, 33 and 34) have been reclassified as belonging to other bacterial species $[8,9]$. The distribution of serotypes recovered from diseased pigs in different geographical regions varies, although serotypes 2 and 9 are the most prevalent in several European countries [1]. Serotype 2 is, by far, the most common serotype affecting humans, followed by serotype 14 [1]. The distribution of serotypes affecting pigs in North America is different, with no clear prevalence of serotype $2[10,11]$. Highly, intermediately and low virulent serotype 2 strains have been characterized $[7,12]$. Further studies using multilocus sequence typing (MLST) showed that sequence type (ST) 1 strains (with other clonal complex 1 strains) normally found in Europe and Asia present higher virulence potential and are mainly isolated from diseased pigs and humans [6].

S. suis is normally present in the tonsils and nasopharynx of most healthy pigs $[13,14]$ and some authors therefore classify the bacterium as "pathobionts" [15]. The conditions under which certain serotypes/strains of S. suis become pathogenic and cross the mucosal barrier into the blood causing a systemic infection are not fully understood [16]. A plethora of potential virulent factors has been described, although there is still a debate surrounding their significance to the pathogenesis of diseases linked to S. suis infection [7]. It is believed that, under some circumstances, S. suis does not act alone and takes advantage of concomitant or previous infections with other pathogens. Indeed, since co-infections have been largely been associated with the increase in clinical disease caused by S. suis, we will critically discuss the data available in the literature that directly or indirectly address the issue. The definitions of co-infection, superinfection and mixed infections are not always clear but were plainly explained in a recent review [17]. We will 
use the term mixed infection when there is an epidemiological link only (detection of more than one pathogen in organs/tissues) and co-infection for in vitro and in vivo studies that specifically address the interaction between S. suis and other pathogens.

\section{Complexity of the transition from infection (or colonization) to clinical disease caused by $S$. suis}

Should S. suis be considered a primary or secondary/ opportunistic pathogen? As mentioned, S. suis is a normal inhabitant of the upper respiratory tract [2]. The presence of potentially virulent strains alone does not guarantee the appearance of clinical signs, the latter observed sometimes in the absence of such strains, as occurs in North America where highly virulent Eurasian strains are seldom isolated [2]. Indeed, high doses of inoculum (mostly with virulent serotype 2 strains) and aggressive non-natural inoculation routes (intraperitoneal or intravenous) or sometimes two simultaneous routes of infection (intranasal and intramuscular) have been used in experimental trials using conventional pigs to reproduce clinical disease [18-21]. On the other hand, mortalities up to $20 \%$ with non-serotype 2 strains may be observed in the natural infection in the field if no medication is used [22]. As mentioned, the definition of virulence for a given strain of $S$. suis is not simple, a fact that is even truer for serotypes other than serotype 2 , which have been less studied and for which animal models to reproduce the disease are almost inexistent. For example, serotype 9 virulent strains must be administrated intravenously to susceptible pigs to reproduce disease [23], even though this serotype is the most prevalent among diseased pigs in several European countries [1]. The situation in North America is even more complicated since serotypes 2 and 9 strains are very different from predominant virulent strains in Europe and display lower virulence $[2,24]$. Indeed, serotype $1 / 2$, a poorly studied non-zoonotic serotype, is the predominant serotype recovered from clinical cases in the USA and Canada [10 and unpublished data]. It has been proposed that, in addition to the potential virulence of the strain(s) present in the herd, several factors may influence the appearance of clinical signs (Figure 1): (a) environmental factors; (b) management factors; (c) host factors and/ or (d) the presence of co-infections. Environmental factors that may influence the appearance of $S$. suis-related diseases include poor ventilation, high humidity, inadequate sanitation, high levels of dust and ammonia and large temperature variations between night and day [2]. Management factors such as high level of cross-fostering,

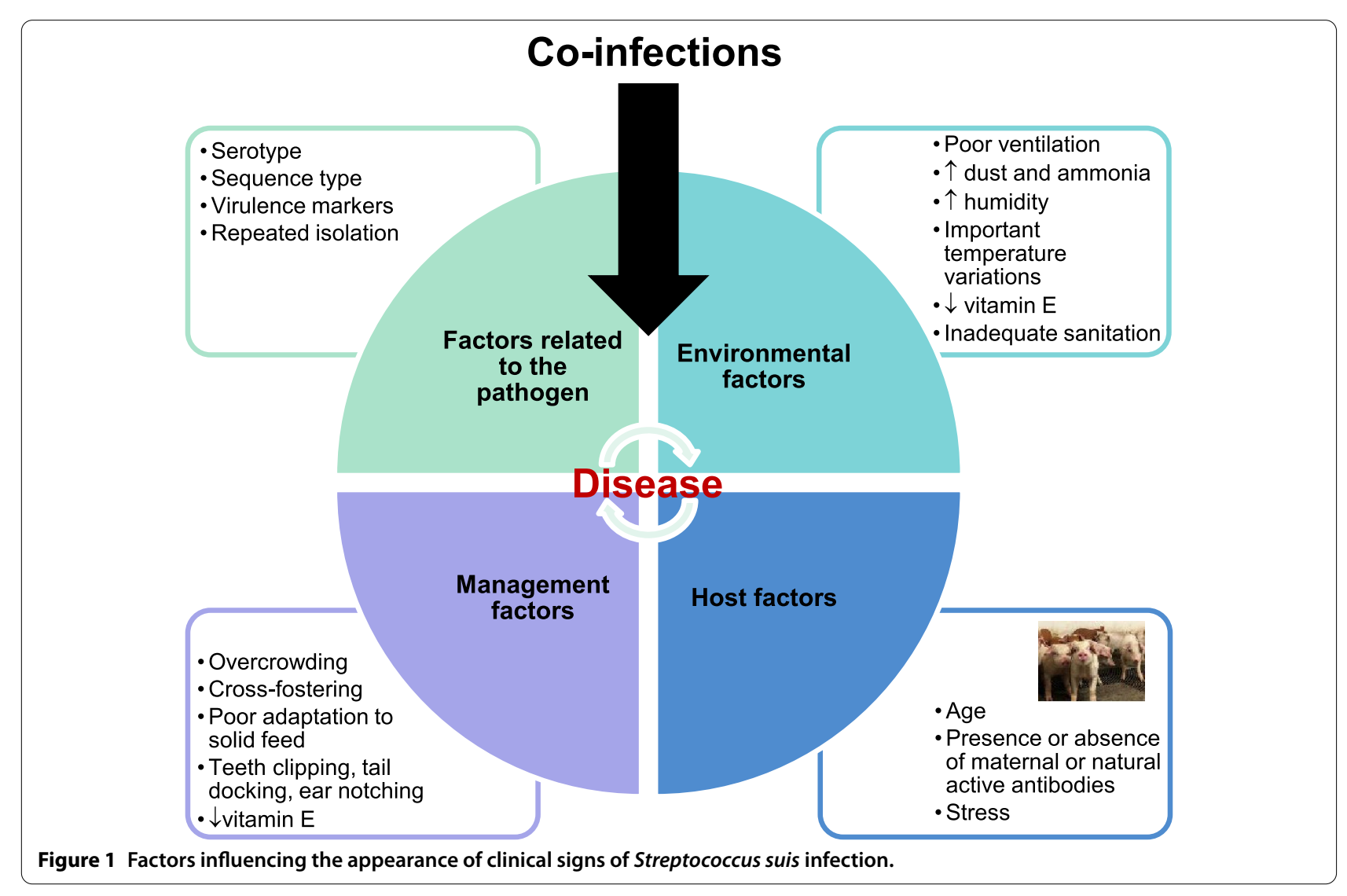


overcrowding, teeth clipping and tail docking, ear notching, mixing pigs of different ages, poor adaptation to solid feed in the nursery and low levels of vitamin $E$ have also been cited as playing important roles [2]. Host factors such as high levels of stress and the presence/absence of anti-S. suis antibodies may also influence the appearance of clinical disease. Indeed, it has been shown that clinical signs appear when the level of maternal antibodies is low [22, 25]; antibodies slowly increase at the end of the post-weaning period, and animals become more resistant to infection [26]. These antibodies would not necessarily be all specific to the virulent strains of S. suis present in the herd (unpublished observations) but probably also to other S. suis or even other streptococci that are normally present in tonsils [25]. Finally, co-infections have been suggested to play a major role in the development of $S$. suis disease. Indeed, co-infections/mixed infections of different swine pathogens with $S$. suis have been reported and suggested in the literature, although there is still a certain lack of scientific support for many of them. This is the main reason why we address the topic in this review.

\section{Should S. suis be considered a respiratory or systemic pathogen? Or both?}

Although many studies addressed the role of S. suis in respiratory diseases, a pathogen's route of entry should be differentiated from the induced pathology. Although there is a proposed hypothesis of intestinal translocation (still to be confirmed) [27], it is widely accepted that the main route of infection for systemic $S$. suis disease is the respiratory route [2], and airborne transmission of the infection has clearly been demonstrated [28]. Within this context, it may be argued whether S. suis should be considered as a true respiratory pathogen. Infectious agents involved in porcine respiratory disease complex (PRDC) are classified into primary or secondary (or opportunistic) pathogens [17]. Primary pathogens are defined as the ones that can infect the animal as the first unique pathogen and then facilitate secondary or opportunistic coinfection(s). S. suis is usually considered as a secondary/ opportunistic pathogen [17]. However, the role of S. suis in respiratory pathology, even as an opportunistic agent, is still not clear. Studies based on natural infection cases indicate that $S$. suis may cause pulmonary lesions generally described as suppurative bronchopneumonia and/or fibrino-hemorrhagic pleuropneumonia [29-31]. However, in most cases of natural infections, other concomitant bacteria were also isolated, and the specific role of $S$. suis is difficult to evaluate [32]. Respiratory clinical signs are usually not observed after experimental infection with S. suis, and the clinical outcomes are mainly septicemia, meningitis and/or arthritis, which are also observed in studied cases of airborne transmission [28, 33]. The few cases in which respiratory signs were observed are frequently associated with heart lesions (endocarditis or pericarditis) with no pulmonary involvement (unpublished observations). Intranasal experimental infections may, under some circumstances (previous irritation of nasal mucosa), induce mostly meningitis and arthritis with no lung lesions associated with respiratory problems ([33] and unpublished data). Intranasal infection of highly susceptible caesarian-derived, colostrum-deprived piglets also led to systemic disease [34]. More recent coinfection studies in conventional pigs (see below) showed some lung lesions following $S$. suis infection, although no indication of the presence of other opportunistic bacterial pathogens in these studies is discussed [20]. Indeed, when evaluating isolates of $S$. suis for its potential virulence (pathotypes), those recovered from lungs are either not included in the study or often considered as possibly opportunistic [10, 35]. S. suis may also reach lungs as post-mortem invasion, and that is one of the causes that disqualify the use of isolates recovered from lungs to produce autogenous vaccines [26].

Finally, S. suis recovered from the lungs of young animals with respiratory signs and pulmonary lesions should be differentiated from those recovered from lungs at slaughter. S. suis rarely induce disease in finisher animals due to the high levels of antibodies present in older animals [25], and the detection of the pathogen in such mixed infections at slaughter should not be taken into consideration. Indeed, S. suis may be isolated from healthy lungs without lesions, probably due to the fact that some carrier isolates may go from the upper to the lower respiratory tract during agony $[2,36]$.

\section{S. suis and viral mixed and/or co-infections}

Mixed infections of $S$. suis with swine viruses are a frequent finding in swine herds. These interactions are believed to have a synergistic effect, resulting in the aggravation of clinical signs and increased mortality. However, few controlled studies on real co-infections have been conducted. The most important viral pathogens that have been suggested to be associated with S. suis disease are porcine reproductive and respiratory syndrome virus (PRRSV) and, to a lesser extent, swine influenza virus (SIV) and porcine circovirus 2 (PCV-2). Studies that consider other viruses are rare. To better understand the importance of S. suis co-infections with swine viruses, we will discuss the main findings from the reported mixed infections, as well as controlled in vitro and in vivo assays that may help elucidate the interactions. The important details and conditions of in vitro and in vivo experimental studies with $S$. suis and viruses are summarized in Tables 1 and 2. 


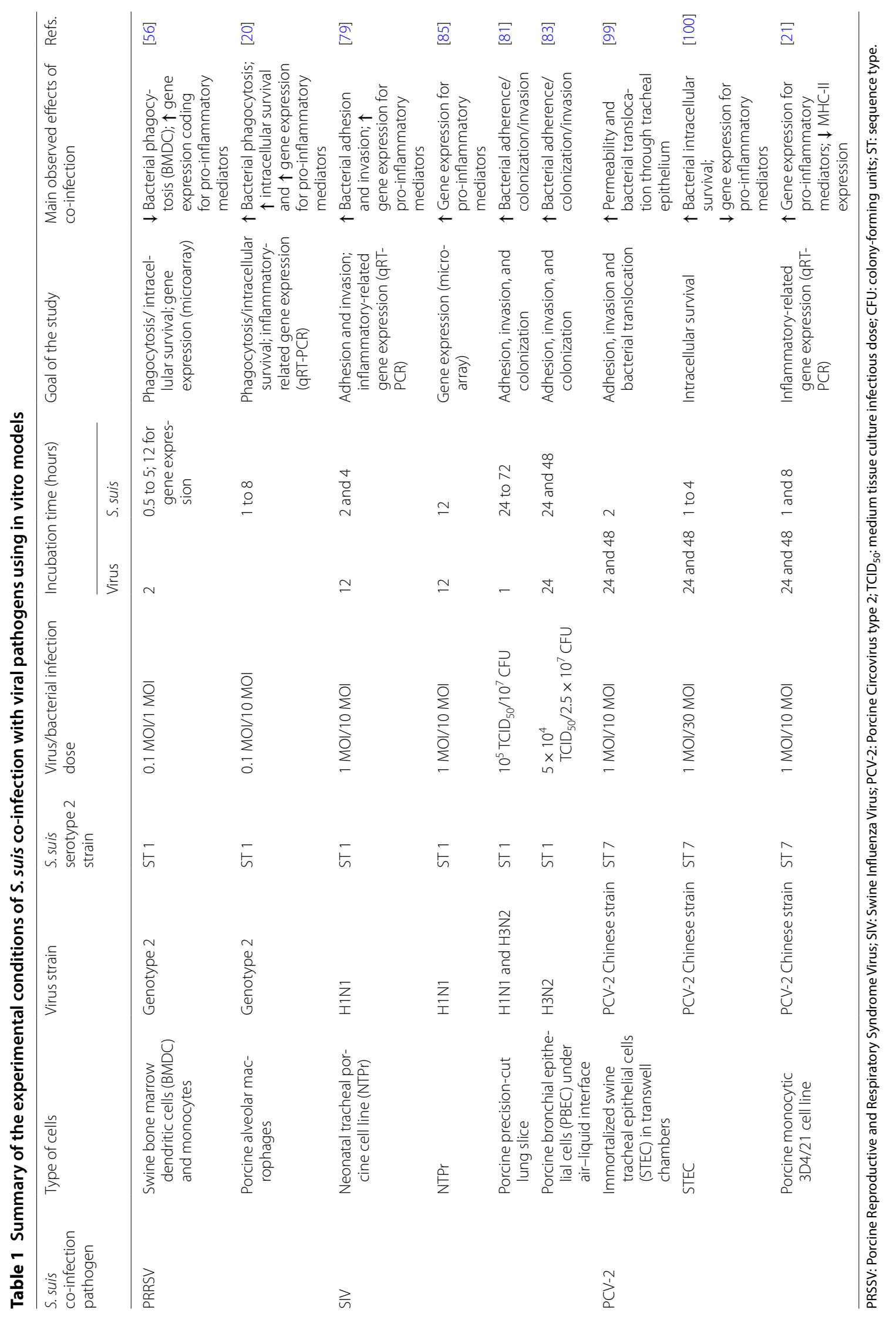




\subsection{S. suis and PRRSV mixed and co-infections}

For over 30 years, PRRSV has been one of the leading viral diseases in pigs, causing significant losses in the swine industry, causing more than $\$ 600$ million in losses every year in the USA alone [37]. The clinical signs can range from reproductive failure in pregnant sows to severe respiratory clinical signs in weaned and growing pigs. PRRSV strains have considerable genetic variability (approximately 60\%) and are categorized as PRRSV1 (European genotype) and PRRSV2 (North American genotype) [38]. PRRSV can cause severe disease alone or act in concert with other viruses or bacteria to significantly contribute to PRDC [40-42]. It has been reported that PRRSV infects pulmonary interstitial, alveolar and intravascular macrophages with consequent decreased phagocytic activity and altered innate immune response in the respiratory tract, opening the door to other opportunistic viral and bacterial pathogens [43]. However, there are still some gaps with regard to the specific cell types the virus infects [37].

\subsubsection{PRRSV-S. suis mixed infections}

There is strong unwritten evidence of a clear synergic association between PRRSV and S. suis from the clinical perspective, and most swine practitioners strongly believe that positive unstable (Category I) herds [44] are more prone to serious problems of $S$. suis disease than PRRSV stable or free herds. Some examples in the literature describe the simultaneous detection of both pathogens in the same farm (mixed infections). Although PRRSV seroprevalence is a good indicator of swine herd exposure to the virus, RT-PCR analysis is required to detect active infections and confirm mixed infections of PRRSV with other swine pathogens [45]. For example, RT-PCR analysis of oral fluids from pig farms in Korea showed that PRRSV and S. suis were frequently detected together (around 50\%) in 3 to 7 week-old piglets [46]. Studies performed at slaughter in Canada showed that RT-PCR analysis of swine pathogens present on tonsils detected S. suis and PRRSV in $53.7 \%$ and $22 \%$ of samples, respectively [13]. Similarly, another study at slaughter showed that lungs that were RT-PCR positive for PRRSV have significantly higher odds of being positive for $S$. suis [47]. These data (from both abattoirs and non-clinical samples) do not reveal the nature of the pathogens' interaction and their possible synergistic effect due to the endemic characteristics of PRRSV and ubiquitous presence of S. suis. Indeed, it is important to state that both pathogens may be present in herds without any associated clinical signs of disease.

In Vietnam, Hoa et al. showed an increased isolation rate of highly virulent $S$. suis serotype 2 in the blood and internal tissues of diseased pigs from PRRSV-affected farms $(18 \%)$ compared to those that were not affected by the virus infection (2\%) [48]. Interestingly, a temporal and spatial association of occurrence of human meningitis caused by S. suis and PRRSV outbreaks in pig farms was observed in that country [49]. It should be noted that the study addresses association and not causality. The study also has limitations (clearly stated by the authors): incomplete details on PRRSV outbreaks, differences in sample collection, underestimation of the real prevalence and distribution of PRRSV outbreaks and lack of details on individual patient data that may constitute confounding factors.

The few epidemiological studies and clinical observations by practitioners around the world point to the importance of the S. suis-PRRSV association with regard to the occurrence of severe disease in pigs. However, the mechanisms behind the observed synergistic effect of both pathogens have yet to be explained. To better understand the cellular, immunological and molecular implications behind this synergy, some in vitro and in vivo models have been developed.

\subsubsection{In vitro studies on the interactions between S. suis and PRRSV}

Owing to the characteristics of PRRSV, S. suis co-infection studies mainly addressed the interactions with cells of the innate immune system. Although the pathogenesis of the infection caused by S. suis is not yet completely understood [7], it is believed that its interaction with respiratory phagocytic cells may be one of the initial steps of the infection [16]. In this first line of defense, pulmonary alveolar macrophages (PAMs) play an essential role in the innate immune response against pathogens through bacterial phagocytosis and elimination [42]. Since these cells are also among PRRSV's most important targets, they may represent an interesting model to study co-infection effects [43]. The hypothesis behind the PAM cells' use in co-infection studies is that a prior PRRSV infection would reduce $S$. suis phagocytosis either by reducing phagocyte activity or inducing cell apoptosis. However, under normal conditions, S. suis possesses a thick capsule that usually prevents bacterial phagocytosis-a fact confirmed by different research groups using nonencapsulated mutants [7]. It is important to note that the bacteria that are internalized do not survive intracellularly [50]. So, it may also be hypothesized that although the internalization of $S$. suis is a rare event, a previous viral infection may affect the intracellular killing of bacteria, which may influence the outcome of the disease. Another important aspect of the interaction of S. suis with phagocytes is the capacity to induce inflammation. Indeed, it has been shown that an excess of inflammation is a hallmark of S. suis disease [7]. Hence, two pathogens 
acting together would increase the inflammatory reaction through an increase in the secretion of pro-inflammatory cytokines. Still, immunosuppression has been demonstrated in some PRRSV infection studies and thus may lead to a higher susceptibility to bacterial co-infection in affected pigs [51, 52]. Interestingly, the two processes are not necessarily in contradiction, since the dual activity of PRRSV (immunosuppression but increased inflammation) has been observed in cases of PRRSV and porcine respiratory coronavirus co-infection studies in pigs [53].

Several studies addressed the interaction between S. suis and PAMs $[50,54,55]$, but only one reported how a previous PRRSV infection of these cells affects S. suis phagocytosis and inflammation (Table 1) [20]. PAMs infected with a highly pathogenic PRRSV genotype 2 strain and a poorly characterized $S$. suis serotype 2 strain showed increased bacterial phagocytosis and survival [20]. In addition, co-infection significantly increased the mRNA expression of most pro-inflammatory cytokines tested, including interleukin (IL)-1 $\beta$, IL-6, IL-8, chemokine ( $\mathrm{C}-\mathrm{C}$ motif) ligand 4 (CCL4), tumor necrosis factor- $\alpha$ (TNF- $\alpha$ ) and interferon- $\beta$ (INF- $\beta$ ). Authors concluded that the deleterious effect of the coinfection was mainly due to excessive inflammation [20]. Surprisingly, no other study addressed the effect of coinfection on these cells.

Another study addressed the effect of co-infection using monocytes and swine bone marrow-derived dendritic cells (BMDC) [56]. Both cell types were first infected with a PRRSV genotype 2 strain and then co-infected with the European (ST1) virulent serotype $2 \mathrm{P} 1 / 7$ strain of $S$. suis (used in virulence studies by most laboratories) [56]. Unlike the previous study with PAMs, results showed that PRRSV significantly reduced the internalization of $S$. suis by the BMDC with no observed effect on bacterial intracellular survival [20]. No differences were observed with monocytes, which hardly allowed virus replication and poorly phagocytosed $S$. suis [56]. As shown with PAMs, microarray analysis revealed significant up-regulation of pro-inflammatory genes in co-infected BMDC [56]. Previous S. suis research showed the capacity of the bacteria alone to induce the secretion of pro-inflammatory cytokines involved in disease pathogenesis [57]. Co-infection with PRRSV may exacerbate the inflammation by increasing the secretion of cytokines from immune cells, although this hypothesis should be confirmed in vivo. Unfortunately, there are no other studies of co-infection of these two important pathogens with phagocytic cells. Indeed, it has been shown that pulmonary intravascular macrophages may play an important role in PRRSV infection [58]. Although a possible association between a virus-dependent suppression of the functions of these cells and an increased susceptibility to S. suis secondary infection has been hypothesized, no study has specifically addressed this interaction. However, an interesting feature of PRRSV is that it may affect the thymus and its ability to carry out its normal functions $[52,59]$. In this way, pigs would be less able to resist and/or eliminate secondary infections. It has also been reported that $S$. suis infection can cause atrophy of the thymus and induce apoptosis of thymocytes, thus likely suppressing host immunity [60]. How these two pathogens interact with this important immune organ is still unknown.

Finally, co-infection studies with non-immune cells have not been conducted. Since PRRSV induces nonsuppurative rhinitis and metaplasia of the turbinate epithelium, it has been suggested that it may predispose pigs to the colonization of the respiratory tract by $S$. suis serotype 2, potentially creating a portal of entry for S. suis [61]. However, the hypothesis of virus predisposition to bacterial colonization has never been confirmed.

In summary, although it is widely accepted that a PRRSV infection increases the susceptibility to $S$. suis coinfection (see results in vivo below), it seems evident that there is a serious lack of scientific evidence that clearly explains the specific mechanisms involved in such interactions and more mechanistic studies are needed.

\subsubsection{In vivo studies on the interactions between $S$. suis and PRRSV}

Although a first report failed to demonstrate any influence of PRRSV infection on an S. suis secondary infection [62], further in vivo experiments were carried out to demonstrate the synergistic effect of these pathogens on morbidity and the severity of clinical signs in pigs [20, $52,61,63,64]$. The most common type of co-infection model was developed by infecting animals intranasally with PRRSV and then, after 5 to 7 days, inoculating $S$. suis intranasally or intramuscularly (an overview of the important study parameters is shown in Table 2) [20, 61, $63,65]$. Only one study addressed an intrauterine infection with PRRSV (sows) followed by S. suis infection in piglets [52] (see below). Different PRRSV types (low to high virulent) and origins (North America or Asia), as well as S. suis serotype 2 (sometimes poorly characterized) strains, were assayed. There is only one study that used a $S$. suis serotype 7 strain [65]. The consensus of these studies is that higher mortality and morbidity were observed in groups in which piglets were co-infected by the two pathogens compared to those infected only with S. suis, independently of the virus genotype and $S$. suis serotype $[20,61,65]$. S. suis was cultured in higher numbers from tissues and blood (bacteremia) of co-infected animals compared to S. suis mono-infected piglets and macroscopic and microscopic lesions in different internal 


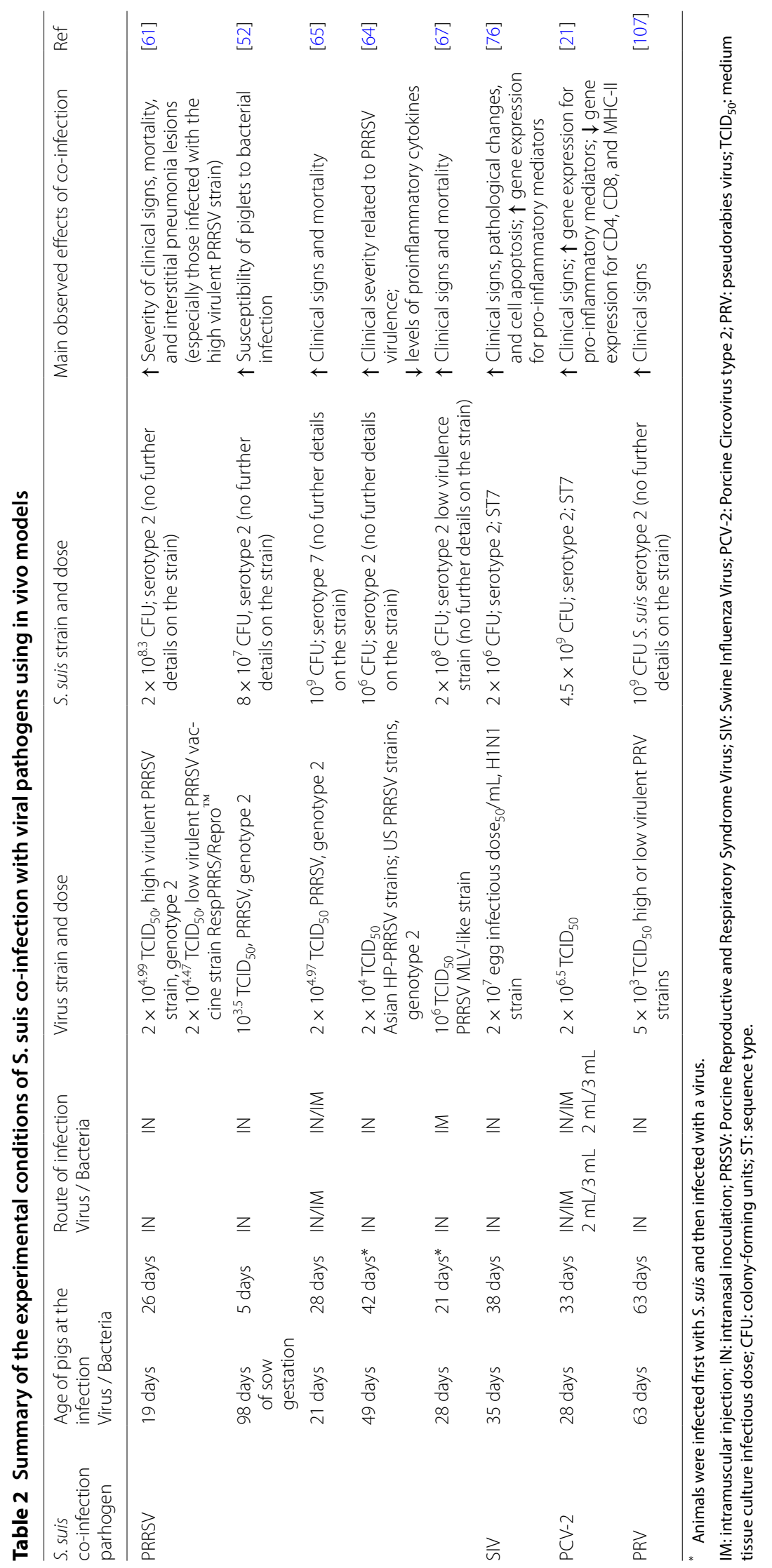


organs were significantly exacerbated in animals in the co-infected groups $[20,61,65]$. In particular, co-infected piglets had significantly more severe gross and microscopic interstitial pneumonia lesions, suggesting a possible secondary role of $S$. suis in PRDC $[20,42,65]$. The co-infection was able to reproduce similar morbidity and mortality to those observed in the high-fever outbreak caused by atypical PRRSV in China [39]. Interestingly, a vaccine strain of PRRSV given intranasally prior to $S$. suis infection induced increased mortality compared to the single-infected controls, although the mortality was lower than in the cases of co-infection with a virulent PRRSV strain [61]. It must be noted that this was an offlabel use of the PRRSV live attenuated vaccine, and it is unlikely that the vaccine would be administered intranasally under field conditions.

PRRSV is a causative agent of complex disease, attacking multiple organs. The infection of pregnant sows with PRRSV results in late abortion, early farrowing and stillborn piglets [66]. Piglets from PRRSV infected sows are PRRSV positive and, in theory, would have a weakened immune system that predisposes them to other opportunistic pathogens [66]. One animal model was developed to examine the effect of intrauterine PRRSV infection in piglets on susceptibility to further intranasal infection with S. suis serotype 2 [52]. To the authors' knowledge, this is the only published study on adult PRRSV infection followed by S. suis piglet co-infection. Pregnant gilts were either inoculated or not with PRRSV at 98 days of gestation (see Table 2) [52]. Five day-old piglets born from these sows were inoculated intranasally with an S. suis serotype 2 strain [52]. The mortality rate, clinical signs, organ lesions and S. suis isolation from joints and brain in the co-infected group were significantly higher compared to control non-S. suis infected piglets and those infected with S. suis alone [52]. Increased susceptibility was explained as being linked to the immuno-suppression caused by the effect of PRRSV on innate immune cells. Indeed, PRRSV-positive piglets had lesions in their thymus and bone marrow and significantly reduced numbers of leukocytes, including lymphocytes and monocytes [52]. In conclusion, this study showed that intrauterine PRRSV infection has significant effects on immune response and may increase the susceptibility of piglets to intranasal S. suis serotype 2 infections. Even so, the presence of confounding factors, especially concerning the experimental model used, must be considered. Piglets were deprived of sow's colostrum, early-weaned and challenged with bacteria at five days of life, which is rare in the field, where mostly post-weaned animals are affected by S. suis $[25,52]$.

Other studies addressed the role of a pre-infection with S. suis with subsequent PRRSV infection. One of these studies used 6-week-old PRRSV-negative piglets weaned before one week of age and kept free of pathogens. Animals were intranasally inoculated with a bacterial cocktail containing S. suis and two other pathogens, mimicking sub-clinical bacterial infections observed in the field. One week later, piglets were challenged with PRRSV strains of different origins and variable virulence [64]. Results showed low mortality in general, although morbidity and lesions were more severe in piglets inoculated with a highly virulent Asian PRRSV strain [64]. The frequency of secondary bacterial pneumonia was directly associated with the clinical severity induced by the PRRSV strains evaluated. Levels of pro-inflammatory cytokines were lower or at the same level in single or co-infected animals, which contradict somehow previous in vitro co-infection studies [20, 56]. Another study used three-week-old conventional piglets that were inoculated intramuscularly with $S$. suis serotype 2 strain and seven days later with a highly pathogenic PRRSV MLV-like isolate [67]. Results showed that while virus or S. suis infection caused transitional fever and moderate clinical signs, the co-infection induced higher fever, anorexia and respiratory distress, leading to $60 \%$ mortality [67]. It should be noted that the route of animal infection with $S$. suis does not reflect natural conditions, and the results should be interpreted carefully.

The synergy between PRRSV and S. suis in vivo cannot be denied, and experimental infection studies generally confirm this fact. However, there are still many variables in previous co-infection studies to identify the mechanisms involved. The pathogenicity of the PRRSV strain is just one part of the puzzle, while other factors like stress, environment, host susceptibility and bacterial burden are some of the variables that may influence the clinical and pathological outcomes. One important point is also the strain of $S$. suis serotype 2 used: in many studies, strains are poorly characterized although it is well known that the virulence of serotype 2 strains is highly variable [7]. One study showed either no effect of co-infection using one S. suis strain (with a North American phenotype) or a strong synergy when a second and different strain (with a Eurasian phenotype) was used $[2,63]$. It is important to note that co-infections of PRRSV and typical North American serotype 2 (ST28 or ST25) S. suis strains are frequently reported in Canada and the USA. Even though the virulence of these $S$. suis strains is probably lower than that of Eurasian strains [7], a combination of other factors may have an influence on the effects of the co-infection (Figure 1). Interestingly, other than the study mentioned above, no co-infection trials have been performed with typical North American S. suis strains. 


\subsection{S. suis and swine influenza type A virus (SIV) mixed and co-infections}

Swine influenza viruses (mainly type A) are able to cause respiratory disease in pigs worldwide, that can lead to $10-15 \%$ mortality [68]. Influenza disease in pigs is highly contagious with no evident clinical signs or mild to moderate ones characterized by runny nose and coughing [69]. Swine influenza causes significant economic losses primarily due to weight loss, though some cases may be much more severe in co-infection with other pathogens, such as M. hyopneumoniae, PRRSV and Actinobacillus pleuropneumoniae [68]. The three main SIV subtypes encountered in pigs are $\mathrm{H} 1 \mathrm{~N} 1, \mathrm{H} 1 \mathrm{~N} 2$, and $\mathrm{H} 3 \mathrm{~N} 2$, and since 2009, the pandemic H1N1 (pH1N1) has also been circulating in domestic pigs worldwide [70]. Influenza viruses cause respiratory disease by infecting the epithelial cells leading to cell apoptosis and the destruction of the mucosal barriers [71]. In addition, cell death is enhanced by the effect of cytokines and innate immune cells, which causes bronchitis and interstitial pneumonia [71].

\subsubsection{SIV-S. suis mixed infections}

It is well known that influenza virus infections in humans are usually complicated by secondary bacterial infections, especially Streptococcus pneumoniae [72]. The capacity of SIV to aggravate respiratory bacterial infections in pigs has also been documented [73-75]. However, the potential of SIV and S. suis mixed or co-infections to cause serious pulmonary disease has been much less studied and there are only a few published reports. Serological data indicate a link between SIV and S. suis in swine farms in China, although detailed information on the correlation of antibody titers and isolation of these two pathogens from healthy or diseased animals is missing [76]. The report on SIV H1N1 virus infections in pigs in England provided more information, since data show that clinical signs were mostly mild with low mortality in older animals infected with SIV alone. Increased mortality was observed in nursery pigs, in which influenza infection was complicated by environmental stress and/ or co-infections, with $S$. suis being the most prevalent pathogen isolated in these cases [77]. S. suis serotypes $1,2,14$, and 24 as well as some untypable isolates were detected in SIV-infected pigs with severe signs of cough, meningitis, lameness and sudden death [77]. These results suggest that SIV may complicate $S$. suis infections observed in the field.

\subsubsection{In vitro studies on the interaction between S. suis and SIV}

Different in vitro and ex vivo tissue cell culture models were therefore developed to examine the complex mechanism of host-pathogen interactions during coinfection (Table 1). Most in vitro studies address the influence of a previous SIV infection on secondary S. suis infection. However, an earlier study using the MadinDarby canine kidney (MDCK) cell line model showed that the supernatant from a S. suis serotype 2 strain can increase the infection ability of SIV H3N2 for these cells [78]. The results may suggest that $S$. suis has secretory factors that facilitate SIV entrance even in the absence of bacteria. It should be noted that MDCK cells do not resemble swine respiratory mucosal cells and, in this study, the effect of the supernatant was evaluated in a closed environment (a well) in which extracellular factors of S. suis are concentrated. The results have not been confirmed or invalidated by other research groups.

The first study that demonstrated the effect of a previous SIV infection in cells followed by a subsequent $S$. suis infection was carried out using neonatal tracheal porcine epithelial (NTPr) cells [79]. SIV H1N1 pre-infected cells enabled bacterial adhesion and invasion levels that were over 100 times higher compared to those of control cells. Inhibition studies confirmed that bacterial capsular sialic acid moiety is responsible for the binding to the viral hemagglutinin expressed on the NTPr cell surface [79]. Also, pre-incubation of S. suis with SIV H1N1 significantly increased bacterial adhesion to epithelial cells and epithelial cell invasion. Similar results were obtained with other S. suis sialic-acid positive serotypes (such as serotypes 1 and 14), but not with serotypes that lack the presence of such sugar moiety in their capsular polysaccharides [79]. The results were confirmed by an independent research group using the same cell type and both SIV H1N1 and H3N2 subtypes [80]. The latter study also revealed that viruses bound to bacteria retained infectivity but induced only tiny plaques compared to the control virus. In contrast, bacterial co-infection had a negative effect on SIV replication. The real cause of this effect is unknown, but the authors hypothesized that the reduction in the amount of released virus may be caused by the direct binding of virions to bacteria and/or a detrimental effect caused by bacteria bound to the surface of infected cells [80]. Further studies are needed to elucidate this effect.

The presence of differentiated epithelial cells and mucus in animal respiratory mucosa creates additional complexity that could not be replicated with epithelial cell lines. To overcome this limitation, Meng et al., established a porcine precision-cut lung slice (PCLS) co-infection model [81]. PCLSs use a procedure that preserves multicellular tissue consisting of ciliated cells, mucusproducing cells and pneumocytes [81]. Hence, the presence of differentiated epithelial cells with preserved functions in this model made it possible to study S. suis 
interactions in an environment that more closely resembles an in vivo respiratory mucosal surface [81]. PLCSs were pre-infected with SIV subtypes H1N1 or H2N3 and subsequently co-infected with an S. suis serotype 2 strain [81]. As shown in previous studies [79, 80], the adhesion of S. suis to epithelial cells was significantly increased by SIV in a bacterial capsule-dependant manner, especially in the early stage [81]. However, at a later stage of coinfection, results were virus strain-dependent, since only SIV H3N2 induced the increased attachment of not only the encapsulated S. suis but also of its non-encapsulated mutant [81]. Indeed, confocal microscopy of cryosections showed the destruction of ciliated epithelial cells and an increased presence of both encapsulated and non-encapsulated S. suis in the sub-epithelium of PLCSs [81]. These results imply that SIV promotes the bacterial infection of respiratory epithelial cells in two phases, depending on viral subtype. The first phase is at the beginning of the infection, when adherence of bacteria is enhanced by viral hemagglutinin (from both virus subtypes) expressed on the surface of the epithelial cells that bind S. suis capsular polysaccharide sialic acid moiety. The second phase occurs later when SIV H3N2 damages the epithelial cells and opens the path for the bacterial colonization of the subepithelial tissue in a capsule (and sialic acid)-independent manner [81].

To better resemble the luminal respiratory environment in airway epithelium, an air-liquid interface (ALI) culture system for differentiated porcine airway epithelial cells was developed [82]. Primary porcine tracheal and bronchial epithelial cells were cultured in a Transwell filter system that enables the differentiation of epithelial cells under air-liquid interface conditions [82]. Indeed, the cell monolayer acquired cilia, pseudostratified epithelium and tight junctions that resemble the cells of airway epithelium in a live animal [82]. The model was first used to evaluate the adhesion and cytotoxic properties of $S$. suis serotype 2, showing that the hemolysin produced by S. suis (suilysin) contributes to the loss of ciliated cells and cell apoptosis, as well as bacterial adhesion and invasion (76). The same system was further developed to examine the interactions of SIV H3N2 and S. suis serotype 2 [83]. The previous infection of cells with the virus increased $S$. suis sialic acid-dependent adherence and colonization, once more confirming previous studies [79-81]. There was a prominent difference in cytopathogenicity observed during the single infection with the bacteria or SIV in this study. S. suis infection resulted in early destruction of the differentiated cells, while SIV H3N2 induced apoptosis in the later stage of infection. It was suggested that $S$. suis cytopathogenicity may be due to the suilysin, since a mutant defective in the production of the toxin adhered but did not damage the cells during the mono-infection. When SIV H3N2 was present in a co-infection study using both wild-type and suilysinnegative mutant strains, the latter was also able to adhere and invade deeper layers of differentiated epithelial cells [83]. Indeed, the authors propose that suilysin-negative S. suis strains, which are common in North America [2], can become invasive in a co-infection scenario with influenza A viruses.

The role of inflammation through an increase of the expression of pro-inflammatory mediators has been described for both S. suis and SIV [84]. A preliminary study showed that co-infection significantly increased the expression of proinflammatory genes [79]. A more detailed study on gene expression in NTPr cells during S. suis and SIV H1N1 co-infection using a microarray assay confirmed that the infection of cells with SIV H1N1 alone or co-infection with both pathogens induced higher mRNA expression of genes in different biological categories than cells infected by S. suis alone, with genes involved in immune response and inflammation being particularly overexpressed [85]. This synergy may be the consequence, at least in part, of an increased bacterial adhesion/invasion of epithelial cells previously infected by SIV.

\subsubsection{In vivo studies on the interaction between S. suis and SIV}

Because $S$. suis and SIV co-infection became of interest just recently, there is only one published animal model (Table 2). Lin et al. developed an in vivo model in which five-week-old piglets were intranasally infected with SIV H1N1 and then intranasally co-infected with a (suilysin positive) S. suis serotype 2 strain three days later [76]. The co-infected group showed more severe clinical signs and viral-induced pneumonia compared to the virus or $S$. suis single-infected groups [76]. The results confirmed, at least in part, the clinical and epidemiological field data from England in which clinical signs seemed to be aggravated in co-infected animals [77]. Interestingly, the viral loads in the lungs were significantly higher in the coinfected group compared to the control group infected with either bacteria or SIV H1N1 alone, thus contradicting previous in vitro observations on a possible inhibitory role of S. suis on virus replication [80]. Moreover, the bacterial load was not increased in the co-infected group compared to the S. suis-infected group [76]. Results also showed significant upregulation changes in genes involved in pro-inflammatory (TLR4, MyD88, IL-17D, IL-6, IL-8 and CCL2) and apoptosis (CASP2, CASP3, BCL2L11, FASLG and TNFRSF8) in the coinfected group compared to the control mono-infected groups [76]. These results confirm previous observations in vitro using tracheal epithelial cells [79]. In sum, the 
study results confirm a synergic clinical and pathological effect of SIV H1N1 and S. suis serotype 2 in pigs. However, from the mechanistic perspective, this study did not characterize the immune cells involved in the generation of mucosal and systemic immune responses.

The characterization of molecular and immunological aspects of co-infections in pigs may also contribute to a better understanding of disease in human medicine [86]. Indeed, as mentioned, human influenza disease is often complicated by secondary pneumonia caused by $S$. pneumoniae, which leads to significant morbidity and mortality around the world [72]. However, the mechanisms involved in this co-infection are also partially known [72].

\subsection{S. suis and porcine circovirus $\mathbf{2}$ mixed and co-infections} Porcine circoviruses (PCVs) single-stranded DNA viruses that belong to the genus Circovirus under the family Circoviridae [87]. Four types of PCVs have been described: PCV-1, PCV-2, PCV-3 and PCV-4 [88]. PCV-2 is, by far, the most important PCV for the swine industry and is found to be associated with multiple clinical manifestations of the disease in pigs referred to as porcine circovirus diseases (PCVD) in Europe and as porcine circovirus associated disease (PCVAD) in North America $[89,90]$. The clinical signs of PCVD/PCVAD were first described as a postweaning multisystemic wasting syndrome (PMWS, currently known as PCV-2-systemic disease, PCV-2-SD) in Canada [91]. PCV-2 was also associated with the porcine dermatitis and nephropathy syndrome and with enteritis [92-94]. However, the most prevalent form detected in the field is as PCV-2 subclinical infections [95].

\subsubsection{PCV-2-S. suis mixed infections}

An analysis of 484 field cases of PCV-2-SD in the USA revealed that most cases were in association with other pathogens and only $1.9 \%$ of investigated cases were caused by PCV-2 alone [96]. PCV-2 was most often detected with PRRSV (51.9\%) and M. hyopneumoniae (35.5\%) [96]. Bacterial septicemia and bacterial pneumonia were detected in $14 \%$ and $7.6 \%$ of the PCV-2-SD cases, respectively [96]. The most prevalent bacteria detected in bacterial septicemia cases was $S$. suis. A similar 2009 study on PCV-2 mixed infections from the same region yielded similar results, with $S$. suis found in around 35\% of PCV-2-SD cases with bacterial septicemia [97]. Furthermore, a qPCR assay was applied in a retrospective epidemiological survey of 121 PCV-2-SD cases from Canadian farms to examine the association between PCV-2 and other swine pathogens [98]. The study revealed that high PCV-2 loads increased the odds ratio of isolating $A$. pleuropneumoniae and $S$. suis serotypes $1 / 2,1,2,3,4$ and 7 from respiratory samples [98], but no clear correlation with disease could be established. Another study detected the presence of both pathogens by qPCR in oral fluid samples [46] but, similarly, no association with the disease was observed. However, the percentage of PCV-2 PCR positive results increased with age, whereas positive results for S. suis showed the opposite, indicating that perhaps both pathogens are not necessarily prevalent at the same time [46]. Taken together, although epidemiological results might suggest a possible role of $S$. suis as a complication of PCV-2-associated diseases, no clear data confirm this hypothesis.

\subsubsection{In vitro studies on the interaction between S. suis and PCV-2}

Only three in vitro co-infection studies have been performed using different cell types to study S. suis and PCV-2 co-infection (Table 1) [21, 99, 100]. The results of the first study, which used poorly characterized immortalized swine tracheal epithelial cells, showed that although no increased adhesion or invasion of $S$. suis serotype 2 was observed, the virus pre-infection decreased the expression levels of ZO-1 and occludin, increased cell permeability and contributed to the translocation of $S$. suis serotype 2 across the tracheal epithelium [99]. Although this in vitro model suggests a possible role of PCV-2 during the initial steps of coinfection by $S$. suis, the level of replication of PCV-2 in tracheal and other respiratory epithelia in pigs is limited, since PCV-2 infects mainly lymphoblast and monocytes (101). Therefore, the impact of the described viral effect in this study is somehow controversial.

In a complementary study by the same research group and using the same cells (with the same limitations), the authors suggested that a previous PCV-2 cell infection would enhance bacterial intracellular survival through a decrease in reactive oxygen species. Moreover, the coinfection of tracheal cells with PCV-2 and S. suis would be responsible for a downregulation of the expression of pro-inflammatory cytokines, which would weaken the host's defensive response [100]. The results, which have yet to be confirmed, must be interpreted carefully since it has been largely reported that inflammation plays a major role in the pathogenesis of PCV-2 infections. Again, these results should be carefully interpreted.

In the third in vitro study, a co-infection of the porcine monocytic cell line 3D4/21 with PCV-2 and S. suis serotype 2 induced a significant upregulation of the expression of pro-inflammatory cytokines as well as TLR2, compared to single-infected cells [21]. The upregulation of TLR4 was mainly induced by PCV-2, whereas the downregulation of MHC-II was observed mostly with $S$. suis [21]. Results may indicate a possible role of the coinfection on increased inflammation and the modulation 
of antigen presentation, although more studies are required to confirm the hypothesis.

\subsubsection{In vivo studies on the interaction between $S$. suis and PCV-2}

There is only one in vivo study using both pathogens (Table 2) [21]. In this model, four-week-old piglets were infected with a PCV-2 strain through intranasal and intramuscular inoculation. Five days later, the animals were intranasally and intramuscularly challenged with a S. suis serotype 2 strain. Compared to single-infected pigs, the co-infected group showed higher temperatures and lower weight gains. The amounts of S. suis in the internal organs of co-infected animals were inconsistently and slightly higher than those in the S. suis-infected group, although the biological significance of the results has yet to be confirmed. Co-infected animals had higher scores of clinical signs and lesions of pneumonia, myocarditis and arthritis compared to single-infected animals [21]. Peripheral blood mononuclear cells collected from co-infected piglets showed higher expression levels of inflammatory cytokines, TLR2 and TLR4 and reduced levels of CD4, CD8 and MHC-II. It was also shown that PCV-2 disrupted the integrity and decreased mRNA and protein levels of tight junctions in the lungs of co-infected animals [99]. Although the authors showed a reduced antibody response against $S$. suis in virus-infected animals, a poorly characterized commercial kit available in China only was used [21]. Currently, there is no widely scientifically accepted test to measure antibodies against S. suis [2]. More studies using other strains and different animal models must be conducted to confirm the results.

\subsection{S. suis and other virus co-infections}

The worldwide distribution and high prevalence of S. suis in pig herds increase the possible interactions with multiple viruses. Co-infections of S. suis with pseudorabies virus (PRV) and Nipah virus have been recorded in the field and experimentally studied in pigs. The common characteristics of S. suis, PRV and Nipah virus are that they invade through the respiratory epithelium, have tropism for neurological tissue and are zoonotic pathogens.

PRV, also known as Aujeszky's disease virus, has a significant economic importance for the pig industry [102]. Infected pigs have respiratory or neurological clinical signs with subsequent high mortality [103]. Pigs are natural hosts, although other domestic and wild animals may also be infected [104], and the disease has zoonotic potential [105]. PRV may be associated with other viral pathogens, such as PCV-2, and may be detected together using PCR in clinically ill pigs and aborted fetuses [106]. It was speculated that due to the prevalence of $S$. suis in pig herds, PRV co-infection can aggravate respiratory and neurological outcomes. In an experimental study, nine-week-old piglets were intranasally inoculated with S. suis and a high or low virulent PRV strain (Table 2). Interestingly, both PRV strains induced more severe $S$. suis clinical signs in co-infected piglets [107]. The biological relevance of this type of co-infection is still not clear since there are no recent experimental or epidemiological data evaluating S. suis and PRV associations. PRV has been eradicated from many countries and, when present, vaccination has been used to successfully prevent Aujeszky's disease in pigs. This may partly explain the reason why research into its association with other pathogens is scarce [101].

Nipah virus is a single-stranded non-segmented RNA virus belonging to the family of Paramyxoviridae [108, 109]. It causes disease in multiple animal species with fruit bats and flying foxes being the main reservoirs $[108,110,111]$. Pigs could be naturally infected, but the infection is often asymptomatic with the virus present in nasal cavities [112]. In experimental studies, young piglets displayed pronounced respiratory clinical signs, while older pigs had predominantly neurological clinical signs $[113,114]$. Nipah virus has significant public health impact, since it causes disease in humans, especially in East and Southeast Asia [115], where human infection caused by S. suis is also predominant [49]. In the case of experimental oronasal infection in six-week-old piglets with Nipah virus, it was observed that the piglets with aggravated respiratory clinical signs had S. suis isolated from the respiratory tissue [116]. This finding should be carefully considered, since bacteria were already present in infected pigs (healthy carriers) and the isolation of $S$. suis was merely accidental [116]. As discussed above, the presence of $S$. suis in the respiratory tissue does not prove the involvement of the bacteria in respiratory disease. Although further studies are required to elucidate the relevance of S. suis and Nipah virus co-infection, there are technical and economic obstacles to develop pig experimental co-infection models. The Nipah virus is a biosecurity level 4 pathogen that requires strict measures and special research facilities that are not widely available.

\section{S. suis mixed and/or co-infections with other bacterial pathogens}

Described mixed swine bacterial infections concern mainly respiratory pathogens involved in the PRDC, such as M. hyopneumoniae, Mycoplasma hyorhinis, A. pleuropneumoniae, Actinobacillus suis, Glaesserella parasuis, Bordatella bronchiseptica, Pasteurella multocida and S. suis [42]. As discussed previously, some are primary pathogens (mainly A. pleuropneumoniae and $M$. hyopneumoniae), while others are considered secondary pathogens (S. suis and G. parasuis) [17, 42]. There are 
very few real co-infection studies of $S$. suis with other bacteria, and most reports address mixed infections.

\subsection{S. suis mixed infections with other bacterial pathogens} One of the main problems of most studies is that mixed infections are described at the abattoir [32, 117]. For primary respiratory pathogens that induce fairly typical lesions and affect grower-finisher animals (such as A. pleuropneumoniae), the studies are appropriate [118]. When available, the combination of serological surveys based on validated antibody detection techniques (for example against $A$. pleuropneumoniae and $M$. hyopneumoniae) at the herd level along with the presence of lesions at slaughter may indicate the pathogens' involvement in the pulmonary pathology of pigs [119]. However, for a secondary respiratory pathogen such as $S$. suis that mainly affect nursery piglets and for which validated serological tests are not available, the studies are far less relevant. As mentioned above, S. suis sometimes colonizes the lungs pre- or ante-mortem without playing any major pathological role in pneumonia $[2,119]$.

Early studies with organs recovered from diseased pigs showed that $S$. suis was frequently isolated in conjunction with A. pleuropneumoniae, P. multocida, E. coli and many other microorganisms, although it is not indicated exactly how many of these cases were from lungs versus other internal organs/tissues [120, 121]. It has been reported that $S$. suis was isolated with many other bacterial pathogens in more than $75 \%$ of lungs displaying fibrino-hemorrhagic pneumonia [122]. S. suis and $P$. multocida were isolated together in almost $50 \%$ of lungs from animals suffering from respiratory clinical signs in China [123]. In addition, there was a reported case of concurrent lung infection of $S$. suis and P. multocida in conjunction with Pneumocystis carinii [124]. Using more sensitive PCR techniques, statistical associations were found in samples from lung tissues between PCR-positive results for $P$. multocida or $A$. pleuropneumoniae and $S$. suis [32]. Interestingly, a case of pneumonia in a sow with mixed infections of S. suis and B. bronchiseptica was also described, although S. suis causing disease in adult animals is extremely rare [125]. Another study indicated that almost $30 \%$ of affected lungs yielded common isolation of these two pathogens [126]. The association of both S. suis and B. bronchiseptica was further studied in in vitro and in vivo models (see below). Interesting, there is only one report indicating a possible correlation between S. suis and $M$. hyopneumoniae in the lungs [127].

The indication of the involvement of $S$. suis in mixed infections as a cause of pulmonary disease may also be found in other animal species. There was a reported case of acute death of a racehorse with clinical signs of pneumonia during transport in Japan [128]. Pasteurella caballi, S. suis and Streptococcus zooepidemicus were isolated from the lungs, indicating that the cause of pneumonia and subsequent death was due to the bacterial mixed infection of the lungs [128]. However, although these bacteria were isolated in high loads from lung and tracheo-bronchial lymph nodes, it was not possible to determine if they were the primary cause or secondary invaders [128]. Furthermore, the transport of the horses was lengthy and during winter months-two factors that may have created major environmental stress that enhanced bacterial growth and susceptibility to infection [128].

The isolation of S. suis with other bacterial pathogens in tissues other than lungs has also been reported. $S$. suis was isolated with $M$. hyorhinis or M. hyopneumoniae from the pericardium [129]. Another study reported mixed infections of G. parasuis and S. suis in cases of polyserositis [130]. Both pathogens were also detected together in more than $30 \%$ of samples, although the tissue/organ of origin (lungs versus internal organs) was not reported [131]. Finally, a case of abortion with dual isolation of S. suis and Arcobacter spp was described [132]. Mixed infections caused by different serotypes of $S$. suis should also be considered, as there have been recorded cases of meningitis and septicemia in a seven-week-old piglet caused by $S$. suis serotype 3 cultured from the spleen and bursa and a $S$. suis serotype 7 recovered from the cerebrospinal fluid [133]. It should be noted that all cases discussed in this section represent sporadic mixed bacterial infections without any clear indication of the specific role of co-infections in the development of disease.

\subsection{In vitro and in vivo studies on the interaction between $S$. suis and other bacterial pathogens}

One in vitro study addressed a co-infection between $S$. suis and G. parasuis using NTPr cells and PAMs (Table 3) [54]. G. parasuis is a causative agent of Glässer's disease in pigs. Similar to S. suis, low virulent strains colonize the upper respiratory epithelial surface of healthy animals, while high virulent strains can cross the epithelial barrier and cause mainly systemic disease and (probably) pneumonia (as a secondary invader) [134]. The disease is characterized by polyserositis and polyarthritis [134]. G. parasuis and $S$. suis infections also share similar clinical signs that frequently represent a challenge in the clinical differential diagnosis. The in vitro model using NTPr cells showed that low and high virulent strains of $S$. suis and G. parasuis have a different pattern of adhesion to and invasion of epithelial cells as single infections [54]. However, co-infection studies show limited in vitro interaction between the two bacterial species, which likely use different host receptors. Although it has been found that 


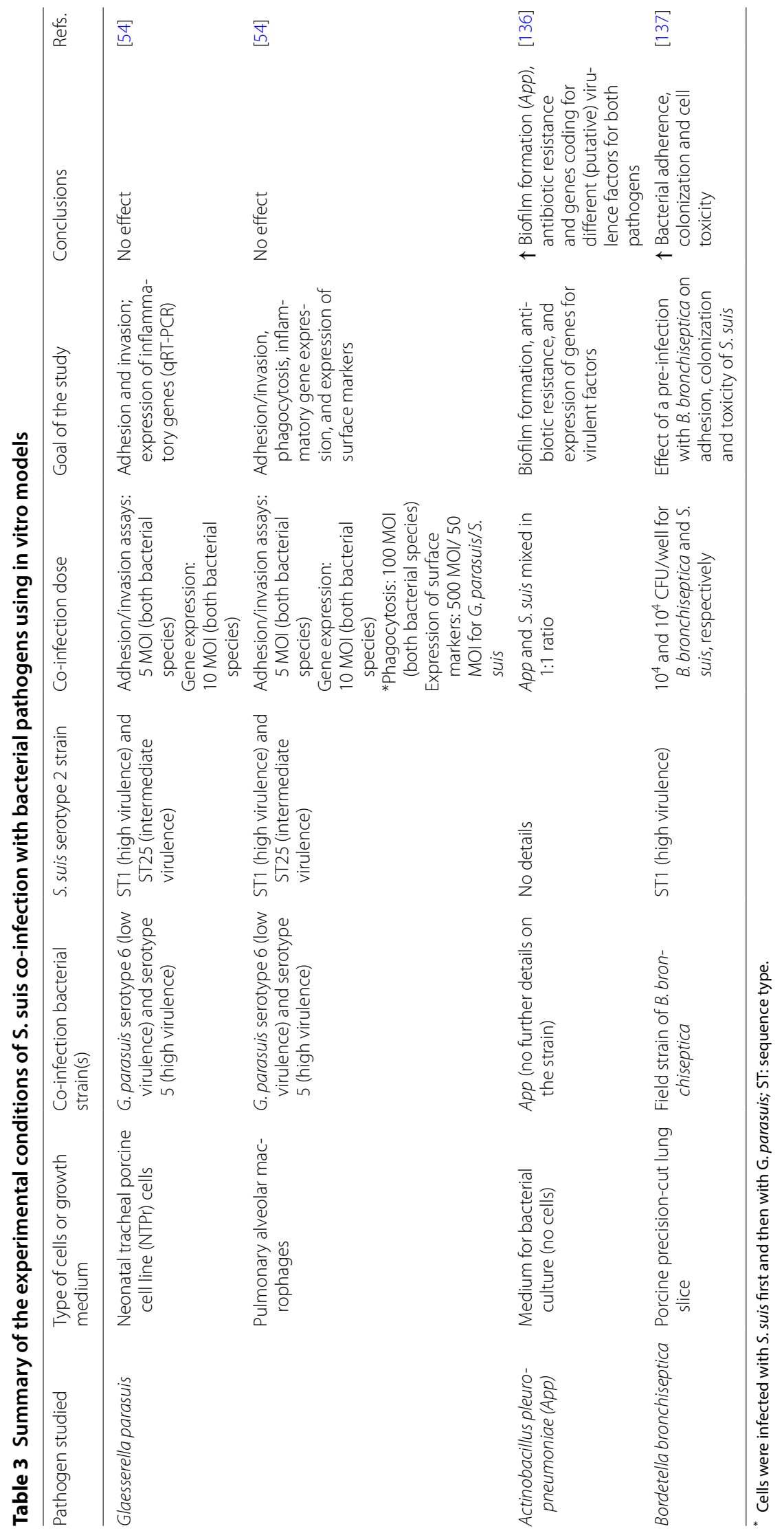


the capsular polysaccharide of S. suis serotype 2 has antiphagocytic properties against a heterologous bacterial species, pre-treatment of PAMs with S. suis did not have a clear effect on the phagocytosis of G. parasuis. When inflammatory mediators produced by both epithelial and PAM co-infected cells were studied, an additive (but not synergistic) effect was observed. Virulence factors of $G$. parasuis and S. suis are not fully known and other conditions in respiratory mucosa may play an important role in G. parasuis and S. suis co-infections [7, 135].

A recent report suggested that co-infections between A. pleuropneumoniae and S. suis are frequently found in China, although no data were presented [136]. However, the authors hypothesized that there are synergistic interactions between both pathogens. They reported that, when A. pleuropneumoniae was cultured with S. suis in culture media, biofilm formation by A. pleuropneumoniae was significantly increased. Moreover, compared to cultures with a single bacterium species, the antibiotic resistance, as well as the expression of genes coding for different (putative) virulence factors of both pathogens, were enhanced in the co-culture model. The authors suggest that the interspecies interactions between $S$. suis and A. pleuropneumoniae may be cooperative under specific conditions and may play an important role in disease progression and persistent infection [136]. It should be noted, however, that both pathogens affect piglets of different ages, with $A$. pleuropneumoniae mainly causing disease in grower-finisher animals and S. suis affecting mostly nursery piglets [36]. Indeed, the implications of the results obtained in the S. suis-A. pleuropneumoniae co-infection study should be carefully interpreted, as they probably do not reflect the situation in the field.

A last in vitro study became available very recently [137], where the interaction of B. bronchiseptica and S. suis was analyzed using the PCLS system previously reported (81). Interestingly, the authors established conditions where the pre-infection of B. bronchiseptica induced reduction of ciliary activity in the absence of significant toxicity. Under these conditions, it was shown that $S$. suis significantly increase adhesion and colonization, although both pathogens seem to adhere and colonize different areas of tissue. In addition, the increased adhesion of S. suis resulted in higher cytotoxicity due to the presence of suilysin [137]. Although not confirmed, in vivo experiments suggest that a pre-infection with $B$. bronchiseptica may promote not only adhesion but also invasion into deeper tissues (see below).

In vivo studies are also very limited (Table 4). There is one report in which S. suis and Aerococcus viridans were isolated together from a pig with meningitis in China [138]. A viridans has been isolated mostly from swine and humans $[139,140]$. However, because $A$. viridans is a bacterium that is present in the environment, it may simply be a contaminant in a sample. To examine the potential of $S$. suis and $A$. viridans interaction, Pan et al. used an intraperitoneal mouse model of co-infection using both bacterial strains recovered from the abovementioned case of meningitis [138]. Results showed that the co-infection significantly increased systemic infection and acute meningitis [138]. Titers of S. suis were significantly increased in the lungs of co-infected mice, while titers of $A$. viridans in lungs remained at a low level [138]. It should be noticed that colonization of the lungs was probably the result of septicemia (due to the injection route) and does not indicate a respiratory infection. Further research is required to see if these results could be reproduced in the swine model and examine the cellular and molecular mechanisms involved in this type of co-infection. However, it is important to keep in mind that his type of co-infection is almost never observed in the field and that the presence of $A$. viridans in the original clinical sample could have simply represented a contamination.

Table 4 Summary of the experimental conditions of S. suis co-infection with bacterial pathogens using in vivo models

\begin{tabular}{|c|c|c|c|c|c|c|c|c|}
\hline $\begin{array}{l}\text { S. suis } \\
\text { coinfection } \\
\text { pathogen }\end{array}$ & $\begin{array}{l}\text { Age of animals } \\
\text { infected with } \\
\text { 1st bacterial spp. }\end{array}$ & $\begin{array}{l}\text { Age of animals } \\
\text { infected with } \\
\text { 2nd bacterial } \\
\text { spp. }\end{array}$ & $\begin{array}{l}\text { 1st bacteria } \\
\text { route of } \\
\text { infection }\end{array}$ & $\begin{array}{l}\text { 2nd bacteria } \\
\text { route of } \\
\text { infection }\end{array}$ & $\begin{array}{l}\text { 1st bacteria and } \\
\text { dose }\end{array}$ & $\begin{array}{l}\text { 2nd bacteria } \\
\text { and dose }\end{array}$ & Conclusions & Refs. \\
\hline $\begin{array}{l}\text { Aerococcus } \\
\text { viridans }\end{array}$ & $\begin{array}{l}\text { Five-week-old } \\
\text { BABL/c mice }\end{array}$ & $\begin{array}{l}\text { Five-week-old } \\
\text { BABL/c mice }\end{array}$ & IP & IP & $\begin{array}{l}10^{3}-10^{8} \text { CFU } \\
\text { S. suis « novel } \\
\text { serotype» (no } \\
\text { further details } \\
\text { on the strain) }\end{array}$ & $\begin{array}{l}10^{3}-10^{8} \mathrm{CFU} \\
\text { A. viridians }\end{array}$ & $\uparrow$ Mortality & [137] \\
\hline $\begin{array}{l}\text { Bordetella bron- } \\
\text { chiseptica }\end{array}$ & $\begin{array}{l}\text { Five-day old } \\
\text { germ-free } \\
\text { piglets }\end{array}$ & $\begin{array}{l}\text { Seven-day-old } \\
\text { germ-free } \\
\text { piglets }\end{array}$ & IN & IN & $\begin{array}{l}0.5 \times 10^{7} \text { CFU } \\
\text { B. bronchiseptica }\end{array}$ & $\begin{array}{l}0.7 \times 10^{6} \mathrm{CFU} ; \\
\text { serotype } 2 \\
\text { ST1, different } \\
\text { phenotypes }\end{array}$ & $\uparrow$ Clinical signs & [141] \\
\hline
\end{tabular}


The most important in vivo study has been carried out with $S$. suis and B. bronchiseptica [141]. Interestingly, the original goal of the use of B. bronchiseptica in this study was simply to predispose pigs to the intranasal infection with $S$. suis. Indeed, as mentioned above, it is difficult to reproduce $S$. suis disease with conventional pigs using this route of infection. In this study, a B. bronchiseptica strain isolated from the nose of a pig with regressive atrophic rhinitis was used. In other words, the study was oriented to a local infection (nose) affecting the mucosa rather than a lower respiratory infection (lungs). Indeed, in further studies, acetic acid was used to replace B. bronchiseptica infection [33]. In the co-infection study, S. suis strains that were phenotypically different with regard to virulence markers were used. Only S. suis strains that harbored the proposed virulence markers were able to induce disease. Interestingly, S. suis was detected in the lungs with bronchopneumonia only when B. bronchiseptica (at higher concentrations) was also present. Indeed, B. bronchiseptica was recovered from the lungs of 19 animals, whereas S. suis was present in 8 samples. None of the samples contained S. suis only. The authors concluded that $S$. suis may be considered a secondary agent of pneumonia [142]. In vitro results recently obtained and discussed above may explain mechanisms involved in the interaction of both bacterial species [137].

The abundance of bacterial pathogens found in epidemiological association with S. suis, especially in pneumonia cases, is not followed by the appropriate number of co-infection studies to understand the role and mechanisms involved in such interactions. In vivo studies of co-infection of $S$. suis with other important bacterial pathogens are not available, and it is therefore difficult to speculate on the significance of each pathogen in the inception of disease.

\section{Limitations of S. suis co-infection studies}

Generally speaking, the limitations of co-infection studies are well addressed by Saade et al. [17]. More specifically with regard to $S$. suis, although mixed infections studies may be taken into consideration, it is important to interpret the data on a real synergic effect in the field carefully, and reports from lungs recovered at the abattoir should be avoided. Indeed, the fact that S. suis is a normal inhabitant of the upper respiratory tract should not be neglected. Regarding in vitro models of co-infection, several have limitations and room for improvement. S. suis infection models using different immortalized or primary cells are still useful to study the mechanisms of gene expression and cellular pathways induced by viruses and bacteria and may shed light on the molecular interactions that occur during co-infections. It should be noted that the choice of cell type and nutritional components of the media may have a major impact on pathogen interactions, growth and survival. For example, the expression of certain metabolic and virulent bacterial genes by $S$. suis may be different or reduced in nutritionally rich media compared to in vivo-like environments [143]. To reproduce cell interactions in a more complex environment, the development of ALI and PCLS systems constitutes a major improvement to study S. suis co-infections [81, 82]. Enhanced in vitro systems such as three-dimensional organotypic tissue models that more closely resemble the 3D architecture, cellular composition and matrix complexity of the organ may be further developed. The adaptability of these tissue-engineered models to multiple pathogens suggests significant potential for infectious disease studies [86].

In many cases, the innate immune system plays an important role in the outcome of the co-infection and, as such, many studies focus on the interaction with the immune cells. In comparison with mouse or human immunology, there is still a lower number of immunological tools designed to detect and analyze swine immune cells [144]. However, since the field of swine immunology is evolving rapidly, we can expect that more sophisticated immunological tools will be developed in the near future and significantly expand our knowledge of the immune response induced during these co-infections.

In general, for many infectious agents, in vivo models of co-infections are sometimes difficult to reproduce [17]. This is even truer when referring to S. suis:

a. Firstly, S. suis is a normal inhabitant and almost all conventional animals used in experimental infections are already colonized. Since there are no sensitive and specific validated tests (neither PCR nor serological tests) to evaluate whether animals are carriers of virulent strains, it is difficult to predict how the animals will behave after experimental infection. We have already recovered $S$. suis serotypes 1 and 14 isolates from the internal organs (one case for each serotype) of animals experimentally infected with a virulent serotype 2 strain, possibly due to a previous infection present in the animals' herds of origin (unpublished observations). On the other hand, the use of caesarian-derived, colostrum-deprived piglets (highly susceptible to $S$. suis) constitutes a very useful tool to generate knowledge but somehow remains relatively far from the reality of the field.

b. Another issue is that the routes of $S$. suis inoculation (even with virulent serotype 2 strains) are rather artificial (intravenous, intraperitoneal, intramuscular) due to the difficulties to reproduce the disease in conventional animals [16]. A seeder-to-naïve model, as developed for other infections such as A. pleuro- 
pneumoniae [145], has yet to be established. In coinfection studies, these procedures complicate the interpretation of the results, since the first pathogen frequently infects animals through the respiratory tract, and both pathogens should be evaluated under similar conditions.

c. As mentioned, the virulence of serotype 2 strains from different geographical origins may be different [2], and almost no co-infection studies were conducted with well-characterized North American serotype 2 strains with lower virulence capacity when compared to their Eurasian counterparts.

d. There are almost no studies with serotypes other than serotype 2 , such as serotype $1 / 2$ which is highly prevalent in North America. This is mainly due to the fact that experimental infection models with nonserotype 2 strains are rarely developed.

e. Environmental and management factors may highly influence the development of the disease by S. suis. These factors are usually not present under experimental conditions in well-controlled research environments. In this regard, recent experiments on $S$. suis mucosal infection models tried to mimic, at least in part, the weaning stress that piglets experience under field conditions by applying several stressors, like transport and social stresses [4].

\section{Conclusion and future perspectives}

Co-infections of $S$. suis with important swine pathogens have been addressed in recent studies, and new mechanisms of pathogen interactions of viral and bacterial pathogens have been proposed. However, owing to the importance given to co-infections in S. suis-associated diseases, the number of studies (mostly addressing mechanisms) is considerably low. In general, viral pathogens such as SIV may destroy the epithelial barrier and increase the permeability of the mucosal surface, while PRSSV may have a negative impact on the functions of pulmonary alveolar and intravascular macrophages and PCV-2 has a strong tropism for lymphoblasts. However, how these mechanisms actually affect $S$. suis pathogenicity is still debatable. Similarly, an increased inflammation (a hallmark of S. suis infections) caused by co-infection with other pathogens may also play an important role. However, most co-infection studies showing upregulations of inflammatory mediators were conducted in vitro and the data must still be validated in vivo within the natural host. Finally, data on co-infections (and mechanisms involved) of S. suis with other bacterial pathogens are mostly unavailable.

Saade et al. made general recommendations for future co-infection studies [17]. These recommendations may be adapted to $S$. suis co-infection studies: (i) authors should clearly indicate and characterize the $S$. suis strains used (e.g., serotype, sequence type, presence of virulence markers and other phenotypic characteristics); (ii) well-characterized S. suis strains of serotypes other than type 2 should also be used, especially in North American studies in which other serotypes (e.g., serotype 1/2) may be more prevalent than serotype 2; (iii) in in vivo studies, the route of infection should follow the natural infection of both pathogens, which should be able to interact in the same environment; (iv) since $S$. suis mainly causes disease in nursery piglets, co-infection studies should mostly address pathogens affecting animals at a similar age; (v) no test is specific or sensitive enough to monitor the status of $S$. suis infection in a herd. Authors should avoid referring to the use of S. suis serotype 2-free animals based on serological (no scientific test available) and/or tonsillar PCR evaluations, being the latter low sensitive. Although some genes involved in the susceptibility or resistance to S. suis disease have been suggested in mice [143], no study has addressed the issue in pigs, and it is therefore not possible to identify animals susceptible to the infection yet; (vi) authors should try to use animals from farms with no or limited clinical cases (and serotype different from the one to be evaluated) of S. suis where no prophylactic/metaphylactic procedures are in place at the nursery; (vii) re-isolation of S. suis, serotyping and, if possible, pathotyping are mandatory in experimental co-infection studies using conventional animals, since $S$. suis other than the strain used during the co-infection may be isolated from diseased animals, especially the lungs. Improved and more standardized models will generate new data on the intricate process of S. suis interactions with major swine pathogens.

Finally, there may be additional effects of a previous infection with a pathogen on a secondary S. suis infection. For example, it has been reported that a previous infection with PRRSV may alter the efficacy of ceftiofur treatment of S. suis co-infection [146]. Whether a previous virus infection affects the antibody and protective response of $S$. suis autogenous vaccines is also unknown [26].

\footnotetext{
Abbreviations

ALI: Air-liquid culture system; BMDC: Bone marrow-derived dendritic cells; DCs: Dendritic cells; MDCK: Madin-Darby canine kidney cells; NTPr: Neonatal tracheal epithelial cells; PAMs: Pulmonary alveolar macrophages; PCLS: Precision-cut lung slice; PCV-2: Porcine circovirus 2; PCVAD: Porcine circovirus associated diseases; PCVD: Porcine circovirus diseases; PMWS: Postweaning multisystemic wasting syndrome; PRDC: Porcine respiratory disease complex; PRRSV: Porcine reproductive and respiratory syndrome virus; PRV: Pseudorabies virus; ST: Sequence type; SIV: Swine influenza virus.
} 


\section{Acknowledgements}

The authors would like to thank the veterinary practitioners in North America and Europe who shared their experiences and clinical observations of co-infection in the field. Research conducted by M.G. and M.S. on S. suis is supported by the Natural Sciences and Engineering Research Council of Canada (NSERC \#04435 to MG, NSERC \#342150 to MS). M.S. holds a Tier 1 Canada Research Chair (CIHR).

\section{Authors' contributions}

All authors equally contributed to the design and drafting of this manuscript. All authors read and approved the final manuscript.

\section{Declaration}

\section{Competing interests}

The authors declare that they have no competing interests.

\section{Author details}

${ }^{1}$ Groupe de Recherche Sur Les Maladies Infectieuses en Production Animale (GREMIP), Centre de Recherche en Infectiologie Porcine et Aviaire (CRIPA), Faculty of Veterinary Medicine, University of Montreal, 3200 Sicotte, Saint-Hyacinthe, QC J2S 2M2, Canada. ${ }^{2}$ UAB, CReSA (IRTA-UAB), Campus de la UAB, 08193 Bellaterra (Cerdanyola del Vallès), Spain. ${ }^{3}$ Departament de Sanitat I Anatomia Animals, Facultat de Veterinària, UAB, 08193 Bellaterra (Cerdanyola del Vallès), Spain. ${ }^{4}$ OIE Collaborating Centre for the Research and Control of Emerging and Re-Emerging Swine Diseases in Europe (IRTA-CReSA), 08193 Bellaterra, Barcelona, Spain.

Received: 15 January 2021 Accepted: 25 February 2021 Published online: 20 March 2021

\section{References}

1. Goyette-Desjardins G, Auger J-P, Xu J, Segura M, Gottschalk M (2014) Streptococcus suis, an important pig pathogen and emerging zoonotic agent-an update on the worldwide distribution based on serotyping and sequence typing. Emerg Microbes Infect 3:1-20

2. Gottschalk M, Segura M (2019) Streptococcosis. In: Diseases of swine, vol 12. Wiley, pp 934-950

3. Seitz M, Valentin-Weigand P, Willenborg J (2016) Use of antibiotics and antimicrobial resistance in veterinary medicine as exemplified by the swine pathogen Streptococcus suis. Curr Top Microbiol Immunol 398:103-121

4. Segura M, Aragon V, Brockmeier SL, Gebhart C, de Greeff A, Kerdsin A, O'Dea MA, Okura M, Saléry M, Schultsz C, Valentin-Weigand P, Weinert LA, Wells JM, Gottschalk M (2020) Update on Streptococcus suis research and prevention in the era of antimicrobial restriction: $4^{\text {th }}$ International Workshop on S. suis. Pathogens 9:374

5. Gottschalk M, Xu J, Calzas C, Segura M (2010) Streptococcus suis: a new emerging or an old neglected zoonotic pathogen? Future Microbiol 5:371-391

6. Wertheim HFL, Nghia HDT, Taylor W, Schultsz C (2009) Streptococcus suis: an emerging human pathogen. Clin Infect Dis 48:617-625

7. Segura M, Fittipaldi N, Calzas C, Gottschalk M (2017) Critical Streptococcus suis Virulence factors: are they all really critical? Trends Microbiol 25:585-599

8. Okura M, Osaki M, Nomoto R, Arai S, Osawa R, Sekizaki T, Takamatsu D (2016) Current taxonomical situation of Streptococcus suis. Pathogens 5:45

9. Tohya M, Sekizaki T, Miyoshi-Akiyama T (2018) Complete genome sequence of Streptococcus ruminantium sp. nov. GUT-187T (=DSM $104980 \mathrm{~T}=\mathrm{JCM} 31869 \mathrm{~T}$ ), the type strain of S. ruminantium, and comparison with genome sequences of Streptococcus suis strains. Genome Biol Evol 10:1180-1184

10. Estrada AA, Gottschalk M, Rossow S, Rendahl A, Gebhart C, Marthaler DG (2019) Serotype and genotype (Multilocus Sequence Type) of Streptococcus suis isolates from the United States serve as predictors of athotype. J Clin Microbiol 57:e00377-e419

11. Gottschalk M, Lacouture, (2015) Canada: Distribution of Streptococcus suis (from 2012 to 2014) and Actinobacillus pleuropneumoniae (from 2011 to 2014) serotypes isolated from diseased pigs. Can Vet J 56:1093-1094

12. Auger J-P, Fittipaldi N, Benoit-Biancamano M-O, Segura M, Gottschalk M (2016) Virulence studies of different sequence types and geographical origins of Streptococcus suis serotype 2 in a mouse model of infection. Pathogens 5:48

13. O'Sullivan T, Friendship R, Blackwell T, Pearl D, McEwen B, Carman S, Slavić D, Dewey C (2011) Microbiological identification and analysis of swine tonsils collected from carcasses at slaughter. Can J Vet Res 75:106-111

14. Baele M, Chiers K, Devriese LA, Smith H, Wisselink H, Vaneechoutte M, Haesebrouck F (2001) The Gram-positive tonsillar and nasal flora of piglets before and after weaning. J Appl Microbiol 91:997-1003

15. Vötsch D, Willenborg M, Weldearegay YB, Valentin-Weigand P (2018) Streptococcus suis - the "two faces" of a pathobiont in the porcine respiratory tract. Front Microbiol 9:480

16. Segura M, Calzas C, Grenier D, Gottschalk M (2016) Initial steps of the pathogenesis of the infection caused by Streptococcus suis: fighting against nonspecific defenses. FEBS Lett 590:3772-3799

17. Saade G, Deblanc C, Bougon J, Marois-Créhan C, Fablet C, Auray G, Belloc C, Leblanc-Maridor M, Gagnon CA, Zhu J, Gottschalk M, Summerfield A, Simon G, Bertho N, Meurens F (2020) Co-infections and their molecular consequences in the porcine respiratory tract. Vet Res 51:80

18. Goyette-Desjardins G, Calzas C, Shiao TC, Neubauer A, Kempker J, Roy R, Gottschalk M, Segura M (2016) Protection against Streptococcus suis serotype 2 infection using a capsular polysaccharide glycoconjugated vaccine. Infect Immun 84:2059-2075

19. Berthelot-Hérault F, Gottschalk M, Morvan H, Kobisch M (2005) Dilemma of virulence of Streptococcus suis: Canadian isolate 89-1591 characterized as a virulent strain using a standardized experimental model in pigs. Can J Vet Res 69:236-240

20. Li J, Wang J, Liu Y, Yang J, Guo L, Ren S, Chen Z, Liu Z, Zhang Y, Qiu W, Li Y, Zhang S, Yu J, Wu J (2019) Porcine reproductive and respiratory syndrome virus NADC30-like strain accelerates Streptococcus suis serotype 2 infection in vivo and in vitro. Transbound Emerg Dis 66:729-742

21. Wang Q, Zhou H, Hao Q, Li M, Liu J, Fan H (2020) Co-infection with porcine circovirus type 2 and Streptococcus suis serotype 2 enhances pathogenicity by dysregulation of the immune responses in piglets. Vet Microbiol 243:108653

22. Cloutier G, D'Allaire S, Martinez G, Surprenant C, Lacouture S, Gottschalk M (2003) Epidemiology of Streptococcus suis serotype 5 infection in a pig herd with and without clinical disease. Vet Microbiol 97:135-151

23. Beineke A, Bennecke K, Neis C, Schröder C, Waldmann K-H, Baumgärtner W, Valentin-Weigand P, Baums CG (2008) Comparative evaluation of virulence and pathology of Streptococcus suis serotypes 2 and 9 in experimentally infected growers. Vet Microbiol 128:423-430

24. Zheng H, Du P, Qiu X, Kerdsin A, Roy D, Bai X, Xu J, Vela Al, Gottschalk M (2018) Genomic comparisons of Streptococcus suis serotype 9 strains recovered from diseased pigs in Spain and Canada. Vet Res 49:1

25. Corsaut L, Misener M, Canning P, Beauchamp G, Gottschalk M, Segura, (2020) Field study on the immunological response and protective effect of a licensed autogenous vaccine to control Streptococcus suis infections in post-weaned Piglets. Vaccines 8:384

26. Rieckmann K, Pendzialek S-M, Vahlenkamp T, Baums CG (2020) A critical review speculating on the protective efficacies of autogenous Streptococcus suis bacterins as used in Europe. Porc Health Manag 6:12

27. Ferrando ML, Schultsz C (2016) A hypothetical model of host-pathogen interaction of Streptococcus suis in the gastro-intestinal tract. Gut Microbes 7:154-162

28. Berthelot-Hérault F, Gottschalk M, Labbé A, Cariolet R, Kobisch M (2001) Experimental airborne transmission of Streptococcus suis capsular type 2 in pigs. Vet Microbiol 82:69-80

29. Erickson ED, Doster AR, Pokorny TS (1984) Isolation of Streptococcus suis from swine in Nebraska. Am Vet Med Assoc 185:666-668

30. Vasconcelos D, Middleton DM, Chirino-Trejo JM (1994) Lesions caused by natural infection with Streptococcus suis type 9 in weaned pigs. J Vet Diagn Investig 6:335-341

31. Reams RY, Glickman LT, Harrington DD, Thacker HL, Bowersock TL (1994) Streptococcus suis infection in swine: a retrospective study of 256 cases. Part II. Clinical signs, gross and microscopic lesions, and coexisting microorganisms. J Vet Diagn Investig 6:326-334 
32. Fablet C, Marois C, Dorenlor V, Eono F, Eveno E, Jolly JP, Le Devendec L, Kobisch M, Madec F, Rose N (2012) Bacterial pathogens associated with lung lesions in slaughter pigs from 125 herds. Res Vet Sci 93:627-630

33. Pallarés FJ, Halbur PG, Schmitt CS, Roth JA, Opriessnig T, Thomas PJ, Kinyon JM, Murphy D, Frank DE, Hoffman L (2003) Comparison of experimental models for Streptococcus suis infection of conventional pigs. Can J Vet Res 67:225-228

34. Brockmeier SL, Loving CL, Eberle KC, Hau SJ, Mou KT, Kehrli ME (2019) Administration of granulocyte-colony stimulating factor (G-CSF) to pigs results in a longer mean survival time after exposure to Streptococcus suis. Vet Microbiol 231:116-119

35. Wileman TM, Weinert LA, Howell KJ, Wang J, Peters SE, Williamson SM, Wells JM, Langford PR, Rycroft AN, Wren BW, Maskell DJ, Tucker AW (2019) Pathotyping the zoonotic pathogen Streptococcus suis: novel genetic markers to differentiate invasive disease-associated isolates from non-disease-associated isolates from England and Wales. J Clin Microbiol 57:e01712-e1718

36. Ruggeri J, Salogni C, Giovannini S, Vitale N, Boniotti MB, Corradi A, Pozzi P, Pasquali P, Alborali GL (2020) Association between infectious agents and lesions in post-weaned piglets and fattening heavy pigs with porcine respiratory disease complex (PRDC). Front Vet Sci 7:636

37. Montaner-Tarbes S, del Portillo HA, Montoya M, Fraile L (2019) Key gaps in the knowledge of the porcine respiratory reproductive syndrome virus (PRRSV). Front Vet Sci 6:38

38. Meng XJ, Paul PS, Halbur PG, Lum MA (1995) Phylogenetic analyses of the putative $M(\mathrm{ORF} 6$ ) and $N(\mathrm{ORF} 7$ ) genes of porcine reproductive and respiratory syndrome virus (PRRSV): implication for the existence of two genotypes of PRRSV in the U.S.A. and Europe. Arch Virol 140:745-755

39. van Geelen A, Anderson TK, Lager KM, Das PB, Otis NJ, Montiel NA Miller LC, Kulshreshtha V, Buckley AC, Brockmeier SL, Zhang J, Gauger PC, Harmon KM, Faaberg KS (2018) Porcine reproductive and respiratory disease virus: Evolution and recombination yields distinct ORF5 RFLP 1-7-4 viruses with individual pathogenicity. Virology 513:168-179

40. Zhou Y-J, Hao X-F, Tian Z-J, Tong G-Z, Yoo D, An T-Q, Zhou T, Li GX, Qiu HJ, Wei TC, Yuan XF (2008) Highly virulent porcine reproductive and respiratory syndrome virus emerged in China. Transbound Emerg Dis 55:152-164

41. Salquero FJ, Frossard J-P, Rebel JMJ, StadejekT, Morgan SB, Graham SP, Steinbach F (2015) Host-pathogen interactions during porcine reproductive and respiratory syndrome virus 1 infection of piglets. Virus Res 202:135-143

42. Opriessnig T, Giménez-Lirola LG, Halbur PG (2011) Polymicrobial respiratory disease in pigs. Anim Health Res Rev 12:133-148

43. Gómez-Laguna J, Salquero FJ, Pallarés FJ, Carrasco L (2013) Immunopathogenesis of porcine reproductive and respiratory syndrome in the respiratory tract of pigs. Vet J 195:148-155

44. Holtkamp DJ, Polson DD, Torremorell M, Morrison B, Classen DM, Becton L, Henry S, Rodibaugh MT, Rowland RR, Snelson H, Straw B, Yeske P, Zimmerman J (2011) Terminology for classifying the porcine reproductive and respiratory syndrome virus (PRRSV) status of swine herds. Tierarztl Prax Ausg G Grosstiere Nutztiere 39:101-112

45. Spagnuolo-Weaver M, Walker IW, McNeilly F, Calvert V, Graham D, Burns K, Adair BM, Allan GM (1998) The reverse transcription polymerase chain reaction for the diagnosis of porcine reproductive and respiratory syndrome: comparison with virus isolation and serology. Vet Microbiol 62:207-215

46. Cheong Y, Oh C, Lee K, Cho K (2017) Survey of porcine respiratory disease complex-associated pathogens among commercial pig farms in Korea via oral fluid method. JVet Sci 18:283-289

47. Zha Y, Xie J, Chen Y, Wei C, Zhu W, Chen J, Qi H, Zhang L, Sun L, Zhang X, Zhou P, Cao Z, Qi W, Zhang M, Huang Z, Zhang G (2013) Microbiological identification and analysis of swine lungs collected from carcasses in swine farms, China. Indian J Microbiol 53:496-498

48. Hoa NT, Chieu TTB, Do Dung S, Long NT, Hieu TQ, Luc NT, Nhuong PT, Huong VT, Trinh DT, Wertheim HF, Van Kinh N, Campbell Jl, Farrar J, Chau NV, Baker S, Bryant JE (2013) Streptococcus suis and porcine reproductive and respiratory syndrome. Vietnam Emerg Infect Dis 19:331-333

49. Huong VTL, Thanh LV, Phu VD, Trinh DT, Inui K, Tung N, Hoa NT, Bryant JE, Horby PW, Kinh NV, Wertheim HF (2016) Temporal and spatial association of Streptococcus suis infection in humans and porcine reproductive and respiratory syndrome outbreaks in pigs in northern Vietnam. Epidemiol Infect 144:35-44
50. Smith HE, Damman M, van der Velde J, Wagenaar F, Wisselink HJ, StockhofeZurwieden N, Smits MA (1999) Identification and characterization of the cps locus of Streptococcus suis serotype 2: the capsule protects against phagocytosis and is an important virulence factor. Infect Immun 67:1750-1756

51. Wang G, Li L, Yu Y, Tu Y, Tong J, Zhang C, Liu Y, Li Y, Han Z, Jiang C, Wang S, Zhou EM, He X, Cai X (2016) Highly pathogenic porcine reproductive and respiratory syndrome virus infection and induction of apoptosis in bone marrow cells of infected piglets. J Gen Virol 97:1356-1361

52. Feng W, Laster SM, Tompkins M, Brown T, Xu J-S, Altier C, Gomez W, Benfield D, McCaw MB (2001) In utero infection by porcine reproductive and respiratory syndrome virus is sufficient to increase susceptibility of piglets to challenge by Streptococcus suis type II. J Virol 75:4889-4895

53. Renukaradhya GJ, Alekseev K, Jung K, Fang Y, Saif LJ (2010) Porcine reproductive and respiratory syndrome virus-induced immunosuppression exacerbates the inflammatory response to porcine respiratory coronavirus in pigs. Viral Immunol 23:457-466

54. Mathieu-Denoncourt A, Letendre C, Auger J-P, Segura M, Aragon V, Lacouture S, Gottschalk M (2018) Limited interactions between Streptococcus suis and Haemophilus parasuis in in vitro co-infection studies. Pathogens $7: 7$

55. de Greeff A, Benga L, Wichgers Schreur PJ, Valentin-Weigand P, Rebel JMJ, Smith HE (2010) Involvement of NF-kappaB and MAP-kinases in the transcriptional response of alveolar macrophages to Streptococcus suis. Vet Microbiol 141:59-67

56. Auray $G$, Lachance C, Wang Y, Gagnon CA, Segura M, Gottschalk M (2016) Transcriptional analysis of PRRSV-infected porcine dendritic cell response to Streptococcus suis infection reveals up-regulation of inflammatory-related genes expression. PLoS One 11:e0156019

57. Lachance C, Gottschalk M, Gerber PP, Lemire P, Xu J, Segura M (2013) Exacerbated type II interferon response drives hypervirulence and toxic shock by an emergent epidemic strain of Streptococcus suis. Infect Immun 81:1928-1939

58. Thanawongnuwech R, Halbur PG, Thacker EL (2000) The role of pulmonary intravascular macrophages in porcine reproductive and respiratory syndrome virus infection. Anim Health Res Rev 1:95-102

59. Wang G, Yu Y, Cai X, Zhou E-M, Zimmerman JJ (2020) Effects of PRRSV infection on the porcine thymus. Trends Microbiol 28:212-223

60. Wang S, Lyu C, Duan G, Meng F, Yang Y, He YuY, X, Wang Z, Gottschalk M, Li G, Cai X, Wang G, (2020) Streptococcus suis serotype 2 infection causes host immunomodulation through induction of thymic atrophy. Infect Immun 88:e00950-e1019

61. Thanawongnuwech R, Brown GB, Halbur PG, Roth JA, Royer RL, Thacker BJ (2000) Pathogenesis of porcine reproductive and respiratory syndrome virus-induced increase in susceptibility to Streptococcus suis infection. Vet Pathol 37:143-152

62. Cooper VL, Doster AR, Hesse RA, Harris NB (1995) Porcine reproductive and respiratory syndrome: NEB-1 PRRSV infection did not potentiate bacterial pathogens. J Vet Diagn Investig 7:313-320

63. Galina L, Pijoan C, Sitjar M, ChristiansonWT, RossowK, Collins JE (1994) Interaction between Streptococcus suis serotype 2 and porcine reproductive and respiratory syndrome virus in specific pathogen-free piglets. Vet Rec 134:60-64

64. Brockmeier SL, Loving CL, Palmer MV, Spear A, Nicholson TL, Faaberg KS, Lager KM (2017) Comparison of Asian porcine high fever disease isolates of porcine reproductive and respiratory syndrome virus to United States isolates for their ability to cause disease and secondary bacterial infection in swine. Vet Microbiol 203:6-17

65. Xu M, Wang S, Li L, Lei L, Liu Y, Shi W, Wu J, Li L, Rong F, Xu M, Sun G, Xiang H, Cai X (2010) Secondary infection with Streptococcus suis serotype 7 increases the virulence of highly pathogenic porcine reproductive and respiratory syndrome virus in pigs. Virol $J$ 7:184

66. Lunney JK, Fang Y, Ladinig A, Chen N, Li Y, Rowland B, Renukaradhya GJ (2016) Porcine Reproductive and Respiratory Syndrome Virus (PRRSV): pathogenesis and interaction with the immune System. Annu Rev Anim Biosci 4:129-154

67. Sun Y-F, Jiang X, Zhang A, Ma J-F, Yu X-X, Li L-A, Yu H (2020) Early infection of Streptococcus suis serotype 2 increases the virulence of highly pathogenic porcine reproductive and respiratory syndrome MLV-like virus in pigs. Res Vet Sci 130:68-72

68. Ma W (2020) Swine influenza virus: current status and challenge. Virus Res 288:198118 
69. Jung K, Ha Y, Chae C (2005) Pathogenesis of swine influenza virus subtype H1N2 infection in pigs. J Comp Pathol 132:179-184

70. Choi YK, Goyal SM, Kang SW, Farnham MW, Joo HS (2002) Detection and subtyping of swine influenza H1N1, H1N2 and H3N2 viruses in clinical samples using two multiplex RT-PCR assays. J Virol Methods 102:53-59

71. Janke BH (2014) Influenza A virus infections in swine: pathogenesis and diagnosis. Vet Pathol 51:410-426

72. Alicino C, ludici R, Alberti M, Durando P (2011) The dangerous synergism between influenza and Streptococcus pneumoniae and innovative perspectives of vaccine prevention. J Prev Med Hyg 52:102-106

73. Palzer A, Ritzmann M, Wolf G, Heinritzi K (2008) Associations between pathogens in healthy pigs and pigs with pneumonia. Vet Rec 162:267-271

74. Fablet C, Marois-Créhan C, Simon G, Grasland B, Jestin A, Kobisch M, Madec F, Rose N (2012) Infectious agents associated with respiratory diseases in 125 farrow-to-finish pig herds: a cross-sectional study. Vet Microbiol 157:152-163

75. Baraldi TG, Cruz NRN, Pereira DA, Galdeano JVB, Gatto IRH, Silva AFD, Panzardi A, Linhares DCL, Mathias LA, de Oliveira LG (2019) Antibodies against Actinobacillus pleuropneumoniae, Mycoplasma hyopneumoniae and influenza virus and their relationships with risk factors, clinical signs and lung lesions in pig farms with one-site production systems in Brazil. Prev Vet Med 171:104748

76. Lin X, Huang C, Shi J, Wang R, Sun X, Liu X, Zhao L, Jin M (2015) Investigation of pathogenesis of H1N1 influenza virus and swine Streptococcus suis serotype 2 co-infection in pigs by microarray analysis. PLoS One 10:e0124086

77. Williamson SM, Tucker AW, McCrone IS, Bidewell CA, Brons N, Habernoll $\mathrm{H}$, Essen SC, Brown IH; COSI, Wood JL (2012) Descriptive clinical and epidemiological characteristics of influenza A H1N1 2009 virus infections in pigs in England. Vet Rec 171:271

78. Wang K, Lu C (2008) Streptococcus suis type 2 culture supernatant enhances the infection ability of the Swine influenza virus H3 subtype in MDCK cells. Berl Munch TierarztI Wochenschr 121:198-202

79. Wang Y, Gagnon CA, Savard C, Music N, Srednik M, Segura M, Lachance C, Bellehumeur C, Gottschalk M (2013) Capsular sialic acid of Streptococcus suis serotype 2 binds to swine influenza virus and enhances bacterial interactions with virus-infected tracheal epithelial cells. Infect Immun 81:4498-4508

80. Wu N-H, Meng F, Seitz M, Valentin-Weigand P, Herrler G (2015) Sialic acid-dependent interactions between influenza viruses and Streptococcus suis affect the infection of porcine tracheal cells. J Gen Virol 96:2557-2568

81. Meng F, Wu NH, Nerlich A, Herrler G, Valentin-Weigand P, Seitz M (2015) Dynamic virus-bacterium interactions in a porcine precision-cut lung slice co-infection model: Swine influenza virus paves the way for Streptococcus suis infection in a two-step process. Infect Immun 83:2806-2815

82. Meng F, Wu N-H, Seitz M, Herrler G, Valentin-Weigand P (2016) Efficient suilysin-mediated invasion and apoptosis in porcine respiratory epithelial cells after streptococcal infection under air-liquid interface conditions. Sci Rep 6:26748

83. Meng F, Tong J, Vötsch D, Peng J-Y, Cai X, Willenborg M, Herrler G, Wu $\mathrm{NH}$, Valentin-Weigand $\mathrm{P}$ (2019) Viral co-infection replaces effects of suilysin on Streptococcus suis adherence to and invasion of respiratory epithelial cells grown under air-liquid interface conditions. Infect Immun 87:e00350-e419

84. Gu Y, Hsu AC-Y, Pang Z, Pan H, Zuo X, Wang G, Zheng J, Wang F (2019) Role of the innate cytokine storm induced by the influenza A virus. Viral Immunol 32:244-251

85. Dang Y, Lachance C, Wang Y, Gagnon CA, Savard C, Segura M, Grenier D, Gottschalk M (2014) Transcriptional approach to study porcine tracheal epithelial cells individually or dually infected with swine influenza virus and Streptococcus suis. BMC Vet Res 10:86

86. Siemens N, Oehmcke-Hecht S, Mettenleiter TC, Kreikemeyer B, ValentinWeigand P, Hammerschmidt S (2017) Port d'entrée for respiratory infections - Does the influenza A virus pave the way for bacteria? Front Microbiol 8:2602

87. Saikumar G, Das T (2019) Porcine Circovirus. Recent Adv Anim Virol 2:171-195

88. Guo Z, Ruan H, Qiao S, Deng R, Zhang G (2020) Co-infection status of porcine circoviruses (PCV2 and PCV3) and porcine epidemic diarrhea virus (PEDV) in pigs with watery diarrhea in Henan province, central China. Microb Pathog 142:104047

89. Segalés J, Allan GM, Domingo M (2005) Porcine circovirus diseases. Anim Health Res Rev 6:119-142

90. Opriessnig T, Meng X-J, Halbur PG (2007) Porcine circovirus type 2 associated disease: update on current terminology, clinical manifestations, pathogenesis, diagnosis, and intervention strategies. J Vet Diagn 19:591-615

91. Harding JC, Clark EG (1997) Recognizing and diagnosing postweaning multisystemic wasting syndrome (PMWS). J Swine Health Prod 5:201-203

92. Harms PA (2002) Three cases of porcine respiratory disease complex associated with porcine circovirus type 2 infection. J Swine Health Prod 10:27-30

93. Rosell C, Segalés J, Ramos-Vara JA, Folch JM, Rodríguez-Arrioja GM, Duran CO, Domingo M (2000) Identification of porcine circovirus in tissues of pigs with porcine dermatitis and nephropathy syndrome. Vet Rec 146:40-43

94. Jensen TK, Vigre H, Svensmark B, Bille-Hansen V (2006) Distinction between porcine circovirus type 2 enteritis and porcine proliferative enteropathy caused by Lawsonia intracellularis. J Comp Pathol 135:176-182

95. Segalés J (2012) Porcine circovirus type 2 (PCV-2) infections: clinical signs, pathology and laboratory diagnosis. Virus Res 164:10-19

96. Pallarés FJ, Halbur PG, Opriessnig T, Sorden SD, Villar D, Janke BH, Yaeger MJ, Larson DJ, Schwartz KJ, Yoon KJ, Hoffman LJ (2002) Porcine circovirus type 2 (PCV-2) co-infections in US field cases of postweaning multisystemic wasting syndrome (PMWS). J Vet Diagn Investig 14:515-519

97. Opriessnig T, Halbur PG (2012) Concurrent infections are important for expression of porcine circovirus associated disease. Virus Res 164:20-32

98. Gagnon CA, Castillo JRE, Music N, Fontaine G, Harel J, Tremblay D (2008) Development and use of a multiplex Real-Time Quantitative Polymerase Chain Reaction assay for detection and differentiation of porcine circovirus-2 genotypes $2 \mathrm{a}$ and $2 \mathrm{~b}$ in an epidemiological survey. J Vet Diagn Invest 20:545-548

99. Wang Q, Zhou H, Lin H, Ma Z, Fan H (2020) Porcine circovirus type 2 exploits JNK-mediated disruption of tight junctions to facilitate Streptococcus suis translocation across the tracheal epithelium. Vet Res 51:31

100. Wang Q, Zhou H, Fan H, Wang X (2020) Co-infection with PCV-2 and Streptococcus suis serotype 2 (SS2) enhances the survival of SS2 in swine tracheal epithelial cells by decreasing reactive oxygen species production. Infect Immun 88:e00537

101. Pérez-Martín E, Rovira A, Calsamiglia M, Mankertz A, Rodríguez F, Segalés J (2007) A new method to identify cell types that support porcine circovirus type 2 replication in formalin-fixed, paraffin-embedded swine tissues. J Virol Methods 2146:86-95

102. Wong G, Lu J, Zhang W, Gao GF (2019) Pseudorabies virus: a neglected zoonotic pathogen in humans? Emerg Microbes Infect 8:150-154

103. Mettenleiter TC (2020) Aujeszky's disease and the development of the marker/DIVA vaccination concept. Pathogens 9:563

104. Cheng Z, Kong Z, Liu P, Fu Z, Zhang J, Liu M, Shang Y (2020) Natural infection of a variant pseudorabies virus leads to bovine death in China. Transbound Emerg Dis 67:518-522

105. Yang X, Guan H, Li C, Li Y, Wang S, Zhao X, Zhao Y, Liu Y (2019) Characteristics of human encephalitis caused by pseudorabies virus: A case series study. Int J Infect Dis 87:92-99

106. Huang C, Hung J-J, Wu C-Y, Chien M-S (2014) Multiplex PCR for rapid detection of pseudorabies virus, porcine parvovirus and porcine circoviruses. Vet Microbiol 101:209-214

107. Iglesias JG, Trujano M, Xu J (1992) Inoculation of pigs with Streptococcus suis type 2 alone or in combination with pseudorabies virus. Am J Vet Res 53:364-367

108. Soman Pillai V, Krishna G, Valiya Veettil M (2020) Nipah virus: past outbreaks and future containment. Viruses 12:465

109. Chua KB, Bellini WJ, Rota PA, Harcourt BH, Tamin A, Lam SK, Ksiazek TG, Rollin PE, Zaki SR, Shieh W, Goldsmith CS, Gubler DJ, Roehrig JT, Eaton B, Gould AR, Olson J, Field H, Daniels P, Ling AE, Peters CJ, Anderson LJ, Mahy BW (2000) nipah virus: a recently emergent deadly paramyxovirus. Science 288:1432-1435

110. Reynes J-M, Counor D, Ong S, Faure C, Seng V, Molia S, Walston J, Georges-Courbot MC, Deubel V, Sarthou JL (2005) Nipah virus in Lyle's flying foxes, Cambodia. Emerg Infect Dis 11:1042-1047 
111. Halpin K, Hyatt AD, Fogarty R, Middleton D, Bingham J, Epstein JH, Rahman SA, Hughes T, Smith C, Field HE, Daszak P (2011) Pteropid bats are confirmed as the reservoir hosts of henipaviruses: a comprehensive experimental study of virus transmission. Am J Trop Med Hyg 85:946-951

112. Kasloff SB, Leung A, Pickering BS, Smith G, Moffat E, Collignon B, Embury-Hyatt C, Kobasa D, Weingartl HM (2019) Pathogenicity of Nipah henipavirus Bangladesh in a swine host. Sci Rep 9:5230

113. Weingartl HM, Albrecht RA, Lager KM, Babiuk S, Marszal P, Neufeld J, Embury-Hyatt C, Lekcharoensuk P, Tumpey TM, García-Sastre A, Richt JA (2009) Experimental infection of pigs with the human 1918 pandemic influenza virus. J Virol 83:4287-4296

114. Middleton DJ, Westbury HA, Morrissy CJ, van der Heide BM, Russell GM, Braun MA, Westbury HA, Halpin K, Daniels PW (2002) Experimental Nipah virus infection in pigs and cats. J Comp Pathol 126:124-136

115. Geisbert TW, Feldmann H, Broder CC (2012) Animal challenge models of Henipavirus infection and pathogenesis. Henipavirus 359:153-177

116. Berhane Y, Weingartl HM, Lopez J, Neufeld J, Czub S, Embury-Hyatt C, Goolia M, Copps J, Czub M (2008) Bacterial infections in pigs experimentally infected with Nipah virus. Transbound Emerg Dis 55:165-174

117. Merialdi G, Dottori M, Bonilauri P, Luppi A, Gozio S, Pozzi P, Spaggiari B, Martelli P (2012) Survey of pleuritis and pulmonary lesions in pigs at abattoir with a focus on the extent of the condition and herd risk factors. Vet J 193:234-239

118. Sassu EL, Bossé JT, Tobias TJ, Gottschalk M, Langford PR, Hennig-Pauka I (2018) Update on Actinobacillus pleuropneumoniae-knowledge, gaps and challenges. Transbound Emerg Dis 65:72-90

119. Wallgren P, Nörregård E, Molander B, Persson M, Ehlorsson C-J (2016) Serological patterns of Actinobacillus pleuropneumoniae, Mycoplasma hyopneumoniae, Pasteurella multocida and Streptococcus suis in pig herds affected by pleuritis. Acta Vet Scand 58:71

120. Touil F, Higgins R, Nadeau M (1988) Isolation of Streptococcus suis from diseased pigs in Canada. Vet Microbiol 17:171-177

121. Higgins R, Gottschalk M, Mittal KR, Beaudoin M (1990) Streptococcus suis infection in swine. A sixteen-month study. Can J Vet Res 54:170-173

122. Reams RY, Harrington DD, Glickman LT, Thacker HL, Bowersock TB (1995) Fibrinohemorrhagic pneumonia in pigs naturally infected with Streptococcus suis. J Vet Diagn 7:406-408

123. Liu H, Zhao Z, Xi X, Xue Q, Long T, Xue Y (2017) Occurrence of Pasteurella multocida among pigs with respiratory disease in China between 2011 and 2015. Ir Vet $J$ 70:2

124. Kim K-S, Jung J-Y, Kim J-H, Kang S-C, Hwang E-K, Park B-K, Kim DY, Kim JH (2011) Epidemiological characteristics of pulmonary pneumocystosis and concurrent infections in pigs in Jeju Island, Korea. J Vet Sci 12:15-19

125. Griffiths IB, Done SH, Hunt BW (1991) Pneumonia in a sow due to Streptococcus suis type II and Bordetella bronchiseptica. Vet Rec 128:354-355

126. Zhao Z, Wang C, Xue Y, Tang X, Wu B, Cheng X, He Q, Chen H (2011) The occurrence of Bordetella bronchiseptica in pigs with clinical respiratory disease. Vet J 188:337-340

127. Nathues H, Kubiak R, Tegeler R, grosse Beilage E (2010) Occurrence of Mycoplasma hyopneumoniae infections in suckling and nursery pigs in a region of high pig density. Vet Rec 166:194-198

128. Hayakawa Y, Komae H, Ide H, Nakagawa H, Yoshida Y, Kamada M, Kataoka Y, Nakazawa M (1993) An occurrence of equine transport pneumonia caused by mixed infection with Pasteurella caballi, Streptococcus suis and Streptococcus zooepidemicus. J Vet Med Sci 55:455-456

129. Buttenschøn J, Friis NF, Aalbaek B, Jensen TK, Iburg T, Mousing J (1997) Microbiology and pathology of fibrinous pericarditis in Danish slaughter pigs. Zentralbl Veterinarmed A 44:271-280

130. Kang I, Kim D, Han K, Seo HW, Oh Y, Park C, Lee J, Gottschalk M, Chae C (2012) Optimized protocol for multiplex nested polymerase chain reaction to detect and differentiate Haemophilus parasuis, Streptococcus suis, and Mycoplasma hyorhinis in formalin-fixed, paraffin-embedded tissues from pigs with polyserositis. Can J Vet Res 76:195-200

131. Cai X, Chen H, Blackall PJ, Yin Z, Wang L, Liu Z, Jin M (2005) Serological characterization of Haemophilus parasuis isolates from China. Vet Microbiol 111:231-623
132. On SLW, Jensen TK, Bille-Hansen V, Jorsal SE, Vandamme P (2002) Prevalence and diversity of Arcobacter spp. isolated from the internal organs of spontaneous porcine abortions in Denmark. Vet Microbiol 85:159-167

133. Johannson LM (2006) Meningitis and septicemia in a 7-week-old piglet caused by dual streptococcal infection. Can Vet J 47:796-798

134. Costa-Hurtado M, Aragon V (2013) Advances in the quest for virulence factors of Haemophilus parasuis. Vet J 198:571-576

135. Boerlin P, Poljak Z, Gallant J, Chalmers G, Nicholson V, Soltes GA, MacInnes JI (2013) Genetic diversity of Haemophilus parasuis from sick and healthy pigs. Vet Microbiol 167:459-467

136. Wang Y, Gong S, Dong X, Li J, Grenier D, Yi L (2020) In vitro mixed biofilm of Streptococcus suis and Actinobacillus pleuropneumoniae impacts antibiotic susceptibility and modulates virulence factor gene expression. Front Microbiol 11:00507

137. Vötsch D, Willenborg $M$, Baumgärtner $W$, Rohde $M$, Valentin-Weigand $P$ (2021) Bordetella bronchiseptica promotes adherence, colonization, and cytotoxicity of Streptococcus suis in a porcine precision-cut lung slice mode. Virulence 12:84-95

138. Pan Z, Ma Y, Ma J, Dong W, Yao H (2017) Acute meningitis of piglets and mice caused by co-infected with Streptococcus suis and Aerococcus viridans. Microb Pathog 106:60-64

139. Martín V, Vela Al, Gilbert M, Cebolla J, Goyache J, Domínguez L, Fernández-Garayzábal JF (2007) Characterization of Aerococcus viridans isolates from swine clinical specimens. J Clin Microbiol 45:3053-3057

140. Rasmussen M (2013) Aerococci and aerococcal infections. J Infect 66:467-474

141. Vecht U, Wisselink HJ, van Dijk JE, Smith HE (1992) Virulence of Streptococcus suis type 2 strains in newborn germfree pigs depends on phenotype. Infect Immun 60:550-556

142. Wu Z, Wu C, Shao J, Zhu Z, Wang W, Zhang W, Tang M, Pei N, Fan H, Li J, Yao H, Gu H, Xu X, Lu C (2014) The Streptococcus suis transcriptional landscape reveals adaptation mechanisms in pig blood and cerebrospinal fluid. RNA 20:882-898

143. Mair KH, Sedlak C, Käser T, Pasternak A, Levast B, Gerner W, Saalmüller A, Summerfield A, Gerdts V, Wilson HL, Meurens F (2014) The porcine innate immune system: an update. Dev Comp Immunol 45:321-343

144. Rong J, Zhang W, Wang X, Fan H, Lu C, Yao H (2012) Identification of candidate susceptibility and resistance genes of mice infected with Streptococcus suis type 2. PLoS One 7:e32150

145. Lechtenberg KF, Shryock TR, Moore G (1994) Characterization of an Actinobacillus pleuropneumoniae seeder pig challenge-exposure model. Am J Vet Res 55:1703-1709

146. Day DN, Sparks JW, Karriker LA, Stalder KJ, Wulf LW, Zhang J, Kinyon JM, Stock ML, Gehring R, Wang C, Ellingson J, Coetzee JF (2015) Impact of an experimental PRRSV and Streptococcus suis co-infection on the pharmacokinetics of ceftiofur hydrochloride after intramuscular injection in pigs. J Vet Pharmacol Ther 38:475-481

\section{Publisher's Note}

Springer Nature remains neutral with regard to jurisdictional claims in published maps and institutional affiliations.

Ready to submit your research? Choose BMC and benefit from:

- fast, convenient online submission

- thorough peer review by experienced researchers in your field

- rapid publication on acceptance

- support for research data, including large and complex data types

- gold Open Access which fosters wider collaboration and increased citations

- maximum visibility for your research: over $100 \mathrm{M}$ website views per year

At BMC, research is always in progress.

Learn more biomedcentral.com/submissions 